
August 1941

\title{
THERMAL EXPANSION OF CLAY BUILDING BRICKS
}

\author{
By Culbertson W. Ross
}

ABSTRACT

The coefficients of thermal expansion of 139 bricks were measured over the range $-10^{\circ}$ to $+40^{\circ} \mathrm{C}\left(14^{\circ}\right.$ to $\left.104^{\circ} \mathrm{F}\right)$. These bricks included 1 sample of sand-lime, 9 of fire-clay, and 61 of clay and shale bricks. The clay and shale bricks represented a wide range in properties and included samples from various districts in the United States.

The coefficients of 87 percent of the clay and shale bricks were between 5 and 7 millionths per ${ }^{\circ} \mathrm{C}$ (2.8 to 3.9 per $\left.^{\circ} \mathrm{F}\right)$. The average coefficient of the clay bricks was 6.0 (3.3), of the shale bricks 6.1 (3.4), and of the fireclay bricks 3.9 millionths per ${ }^{\circ} \mathrm{C}\left(2.2\right.$ per $\left.^{\circ} \mathrm{F}\right)$. No relation was observed between the thermal expansion and the other physical properties of the bricks.

\section{CONTENTS}

Page

I. Introduction

II. Description of apparatus

III. Test procedure 202

IV. Accuracy of the measurements_.

V. Experimental results___ 213

VI. Conclusions _... 215

\section{INTRODUCTION}

The change in length of building bricks due to temperature changes is one of the factors sometimes blamed for cracking of brick-masonry walls. Inasmuch as a better knowledge of the thermal expansion of bricks should be useful in the study of this and other problems of structural design, measurements have been made of the linear thermal expansion of 139 bricks, representing 1 lot of sand-lime, 9 of fire-clay, and 61 of clay and shale bricks. These bricks, representing the product of various localities in the United States, were available from other investigations and tests and comprised bricks having a wide range in properties. The coefficients of expansion were measured over the temperature range $-10^{\circ}$ to $+40^{\circ} \mathrm{C}\left(14^{\circ}\right.$ to $\left.104^{\circ} \mathrm{F}\right)$, or approximately the range to which they might be subjected in structures. The other physical tests which are customarily used as a measure of quality have been made on the bricks as a matter of record and in the hope that there might be some relation between them and the thermal expansion.

Previous tests of the thermal expansion of bricks have usually been made in connection with the study of some other property of the bricks and are not very extensive.

The Watertown Arsenal report of 1896 gives the thermal expansion of 22 clay and shale bricks over the temperature range $33^{\circ}$ to $212^{\circ} \mathrm{F}$ 
(approx. $0^{\circ}$ to $100^{\circ} \mathrm{C}$ ). The bricks were kept immersed in water, and the changes in length were measured by means of a 6 -inch strain-gage reading to 0.0001 inch. The coefficients of thermal expansion ranged from 3.7 to 13.6 millionths $\mathrm{per}^{\circ} \mathrm{C}\left(2.1\right.$ to $\left.7.6 \mathrm{per}{ }^{\circ} \mathrm{F}\right)$, with an average of $6.3(3.5)$. Fifty percent were within the range 5 to 7 (2.8 to 3.9$)$.

Palmer ${ }^{1}$ reported tests on six types of clay and shale and two types of fire-clay bricks. A 20-inch Whittemore strain gage was used to measure the length changes in specimens consisting of three bricks cemented together with litharge-glycerin cement. The bricks were measured in a cold room at $-8^{\circ} \mathrm{C}$ and in another room at $+25^{\circ} \mathrm{C}$. $\left(17.6^{\circ}\right.$ and $\left.77^{\circ} \mathrm{F}\right)$. Bricks of each type were divided into two classes, one harder burned than the other. The thermal expansion of the harder burned bricks was in general somewhat less than that of the softer bricks. The coefficients of thermal expansion of the clay and shale bricks were between 3.9 and 8.5 millionths per ${ }^{\circ} \mathrm{C}$ (2.2 to 4.7 per ${ }^{\circ} \mathrm{F}$ ), with an average of 5.6 (3.1). The coefficient for the fireclay bricks was about 3.7 millionths per ${ }^{\circ} \mathrm{C}\left(2.1 \mathrm{per}^{\circ} \mathrm{F}\right)$.

Ingberg and Foster ${ }^{2}$ reported thermal-expansion measurements on 16 hollow clay tile, a material somewhat similar to clay bricks. These measurements were made by the interferometer method and over a much larger temperature range than used in the present investigation. From $0^{\circ}$ to $300^{\circ} \mathrm{C}\left(32^{\circ}\right.$ to $\left.572^{\circ} \mathrm{F}\right)$, the coefficients ranged from 5.1 to 7.3 millionths per ${ }^{\circ} \mathrm{C}\left(2.8^{\circ}\right.$ to $\left.4.1 \mathrm{per}^{\circ} \mathrm{F}\right)$ for the 10 clay and shale tile, with an average of 6.2 (3.4). The range of the coefficients of the six fire-clay tile was from 3.5 to 6.8 millionths per ${ }^{\circ} \mathrm{C}\left(1.9^{\circ}\right.$ to 3.8 per $\left.^{\circ} \mathrm{F}\right)$, with an average of 5.5 (3.1).

\section{DESCRIPTION OF APPARATUS}

The Tuckerman optical strain gage ${ }^{3}$ was used to make the thermalexpansion measurements in this investigation. The thermal expansion of the gage was determined by noting the change of the reading with a change in temperature when the gage was mounted on a metal bar, which had been measured from $-10^{\circ}$ to $+40^{\circ} \mathrm{C}$ in the thermalexpansion section of this Bureau. In the same way, the change in the reading between different temperatures with the gage mounted on a brick gave the difference between the thermal expansion of the gage and brick, and thus the thermal expansion of the brick.

A diagram of the Tuckerman optical strain gage, which also shows the method by which it is mounted on a brick, is given in figure 1 . The gage operates on the optical lever principle. A rotation of the stellite lozenge produced by a relative change in length between the gage and the specimen causes the image of the fiduciary point to move on the scale in the autocollimator. The gage is sensitive to a length change of 0.000002 inch. For many of the tests a 4 -inch extension bar was added to the gage, giving a 6-inch gage length. For samples which were available only as half bricks, it was necessary to use the 2-inch gage, as shown in figure 1 .

The knife-edges of the gages could not be placed directly on the bricks as these contain hard minerals, which would cause wear and

1 L. A. Palmer, Volume changes in brick masonry materials, BS J, Research 6, 1003 (1931) RP321.

2 S. H. Ingberg and H. D. Foster, Fire resistance of hollow load-bearing tile, BS J. Research 2, 1 (1929) RP37.

3 L. B. Tuckerman, Optical strain gages and extensometers, Proc. Am. Soc. Testing Materials 23, pt. 2, 602 (1923). 
change the lever arm of the stellite lozenge. Iron plates about 0.1 inch in width were cemented to the bricks with cellulose nitrate cement to provide bearings for the knife-edges. The gage was held on the specimen by a brass ring and screw.

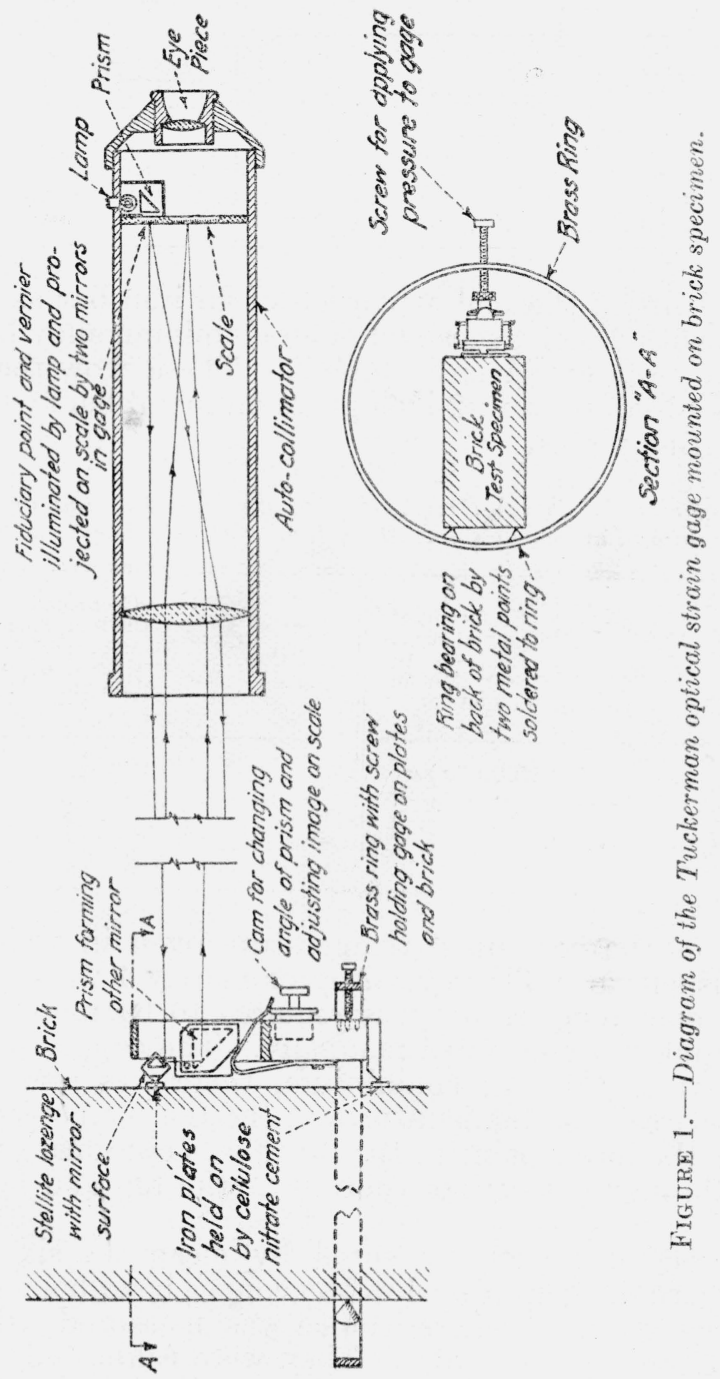

Numerous tests were made, from time to time, on calibrated bars to check the constancy of the gages. The thermal expansion of a 6 -inch gage, as determined by seven tests on such a bar, is given in table 1 . 
TABLE 1.-Coefficient of thermal expansion of 6-inch gage $H$, as determined by seven tests on a calibrated bar over a period of $2 \frac{1}{2}$ years

\begin{tabular}{|c|c|}
\hline Date & $\begin{array}{l}\text { Coefficient of } \\
\text { thermal expan- } \\
\text { sion, }-10^{\circ} \text { to } \\
+40^{\circ} \mathrm{C}\end{array}$ \\
\hline $\begin{array}{l}\text { June } 14,1937 \\
\text { August } 12,1937 \\
\text { February } 3,1938 \\
\text { February } 8,1938 \\
\text { June } 9,1938 \\
\text { August } 31,1939 \\
\text { February } 17,1940\end{array}$ & $\begin{array}{c}\text { Millionths per }{ }^{\circ} \mathrm{C} \\
11.78 \\
11.76 \\
11.78 \\
11.78 \\
11.81 \\
11.77 \\
11.79\end{array}$ \\
\hline
\end{tabular}

The coefficients of thermal expansion of an iron bar as determined by four tests with 2-inch gages and of an aluminum alloy bar as determined by two tests are given in table 2. These show the reproducibility of data obtained with 2-inch gages on metal bars which did not require mounting plates.

TABLE 2.-Coefficient of thermal expansion of an iron bar and of an aluminum alloy bar, as determined by tests with 2-inch gages

\begin{tabular}{|c|c|c|c|}
\hline Gage & $\begin{array}{l}\text { Coefficient of } \\
\text { thermal expan- } \\
\text { sion, }-10^{\circ} \text { to } \\
+40^{\circ} \mathrm{C} \text {, of an } \\
\text { iron bar }\end{array}$ & Gage & $\begin{array}{c}\text { Coefficient of } \\
\text { thermal expan- } \\
\text { sion, }-10^{\circ} \text { to } \\
+40^{\circ} \mathrm{C} \text {, of an } \\
\text { aluminum alloy } \\
\text { bar }\end{array}$ \\
\hline $\begin{array}{l}D \\
E \\
F \\
G\end{array}$ & $\begin{array}{r}\text { Millionths per }{ }^{\circ} \mathrm{C} \\
11.38 \\
11.43 \\
11.36 \\
11.38\end{array}$ & $D_{-}$ & $\begin{array}{c}\text { Millionths per }{ }^{\circ} \mathrm{C} \\
22.31 \\
22.33\end{array}$ \\
\hline
\end{tabular}

A sketch of the controlled temperature oven in which the tests were made is shown in figure 2. Approximately 1 watt per ${ }^{\circ} \mathrm{C}$ elevation above room temperature was required to balance the heat loss. The heat input was controlled manually. The copper plates distributed the heat so that, at equilibrium, there was less than $0.1^{\circ} \mathrm{C}$ difference between the temperatures of the thermometers. The temperature for the lower point of the range was obtained by circulating cold brine through the copper tubes near the top and bottom of the oven.

The temperatures were measured by means of six copper wire resistance thermometers placed in the copper box about the bricks in the locations shown. Their resistance was measured with a Wheatstone bridge and galvanometer. They were calibrated to $0.1^{\circ} \mathrm{C}$ by means of a six-junction thermocouple and a portable potentiometer after being placed in the oven. The calibration was checked on several later occasions and showed no change as great as $0.1^{\circ} \mathrm{C}$.

The gages were read with the autocolimator through holes in the cover of the oven. These holes were normally closed by wooden plugs. The removal of the plugs did not allow enough air to circulate from the room into the oven to cause an observable change in the reading of the gage in the few seconds required for the measurement. 

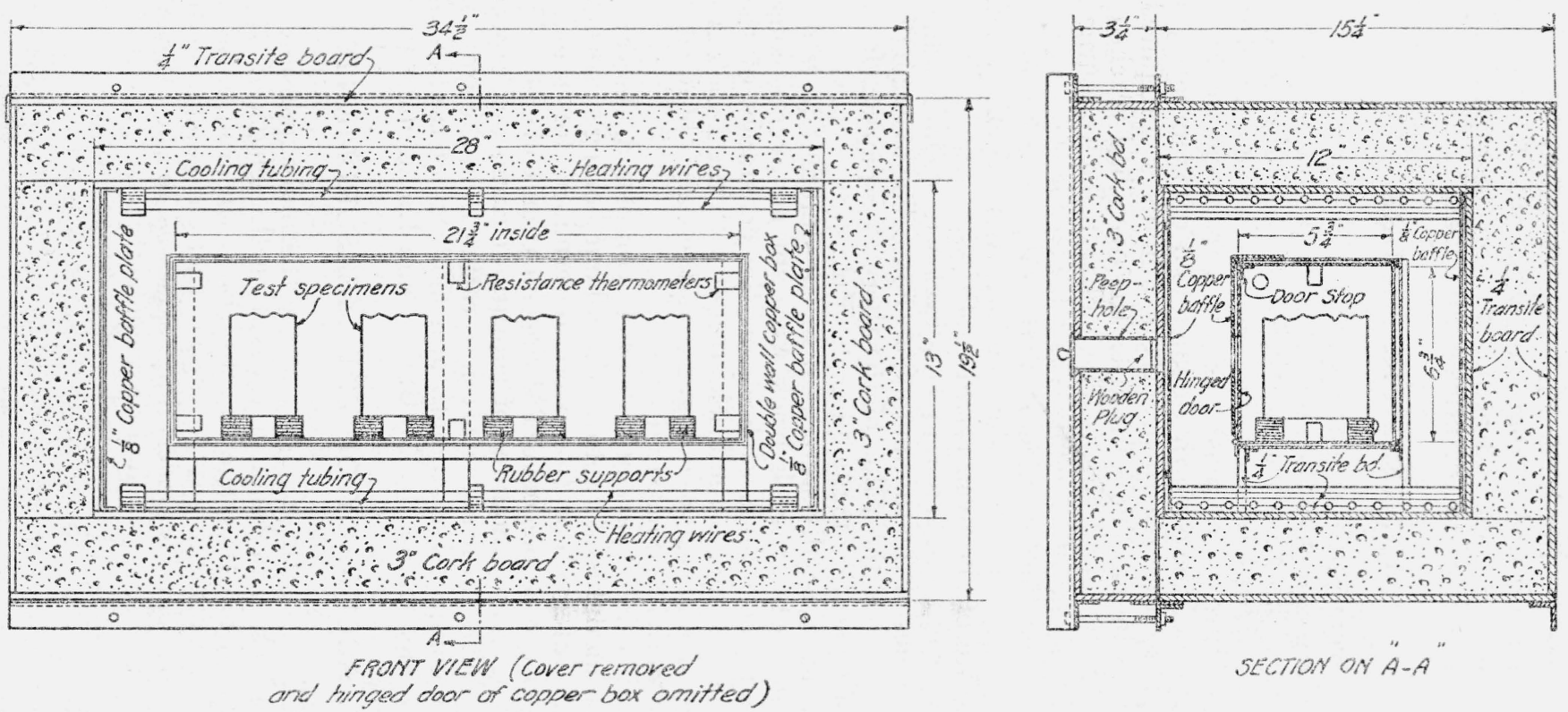

FIGURE 2.-Diagram of the oven in which the thermal expansion tests were made. 
The bricks were supported in the oven by small pieces of rubber cut from automobile tire inner tubes, which allowed the position of the bricks to be adjusted so that the gages could be viewed through the openings in the oven and helped to prevent vibration from the floor being transmitted to the bricks and gages.

\section{TEST PROCEDURE}

The bricks were dried at a temperature of $105^{\circ}$ to $110^{\circ} \mathrm{C}$ before the tests. The gages were then mounted on the bricks and the bricks and gages placed in the oven and their position adjusted so that the gages could be viewed through the openings in the door of the oven.

The temperature of the oven was raised to about $40^{\circ} \mathrm{C}$ and, after the temperature of the oven thermometers became uniform, the readings of the gages were recorded. About 5 hours was required for the temperature to become uniform after the oven had reached the approximate temperature desired. The cooling brine was turned on and the overnight period of 17 hours was allowed for the temperature of the oven to reach the lower point of the range and become uniform, usually at about $-10^{\circ} \mathrm{C}$. The readings were again recorded and the temperature of the oven raised to $40^{\circ} \mathrm{C}$.

The bricks were subjected to two cycles of cooling and heating, giving four values of the thermal expansion. The average of the four values was taken as the value of the thermal expansion for the test. A number of tests were made on each brick.

Different gages usually were used in the different tests of a given brick and the tests on each brick were spread over a considerable period of time as a further check on the constancy of the gages. More tests were made on the bricks tested with the 2 -inch gages than on those tested with the 6 -inch gages as the data obtained with the shorter gage were less consistent.

After the thermal-expansion tests were completed, the compressive strength, the modulus of rupture, the 5-hour boiling absorption, and the 24-hour water absorption were determined for the bricks in accordance with methods of ASTM Specification C67-39. ${ }^{4}$ The compressive strength and modulus of rupture of cored bricks were computed on the basis of the actual sections rather than the gross measurements. The compressive strength and modulus of rupture of bricks 19 to 40 were determined by McBurney and Richmond. ${ }^{5}$

\section{ACCURACY OF THE MEASUREMENTS}

The chief limit on the accuracy of the thermal-expansion measurements was a lack of precision in estimating the length of the portion of the bricks within the gage length. Both the iron plates and the cellulose nitrate cement have a higher thermal expansion than the bricks, and considerable uncertainty exists as to the effective point of attachment. The 0.1 inch of the gage length occupied by the plates amounts to 5 percent of the 2 -inch and 1.7 percent of the 6 -inch gage length. Discrepancies between the values of the thermal expansion from different tests on the same brick were frequently as large as

4 Am. Soc, for Testing Materials Standards, pt. 2, 104 (1939).

5 J. W. MeBurney and J. C. Richmond-Strength, Absorption, and Resistance to Laboratory Freezing and Thawing of Building Bricks Produced in the United States, Building Materials and Struetures (1940), NBS Report BMS60. 
this. However, as most of the tests did not give values that differed by these amounts, and since the average of the values from several tests was taken to obtain the final value, it is believed that the error in the final value for the thermal expansion of a brick is substantially less than 1.7 percent for the data obtained with the 6 -inch gages and 5 percent with the 2 -inch gages. The variation between different tests on metal bars which did not require the mounting plates was much less, as may be seen from table 2 .

After the oven temperature was changed, considerable time was required for the temperature of the bricks to approach that of the oven. Since a substantial difference between the temperature of the bricks and that of the gages at the time readings are made might be expected to introduce an error, the time required for the temperatures to approach equilibrium was checked by placing a resistance thermometer in a hole drilled in the center of one of the bricks and comparing its temperature with that of the oven thermometers and the readings of the gages. These data, which were obtained while the temperature was being raised, are given in table 3 . In this table the apparent coefficients of thermal expansion of the bricks have been computed, for the conditions at each time interval after 30 minutes, from the change in the readings of the gages and the change in temperature of the oven thermometer which indicated the lowest temperature. This thermometer was located at the center of the bottom of the copper box, and from its location would be expected to give a better indication of the temperature of the bricks than any of the other five shown in figure 2 .

TABLE 3.-Changes in the temperature of the oven thermometers, in the temperature of one of the bricks, and in the readings of the gages as the temperature of the oven was raised fram the lower to the higher point of the range

\begin{tabular}{|c|c|c|c|c|c|c|c|}
\hline $\begin{array}{l}\text { Time after heat was } \\
\text { turned on (min) }\end{array}$ & $\begin{array}{c}\text { Highest } \\
\text { of } 6 \text { oven } \\
\text { ther- } \\
\text { mome- } \\
\text { ters }\end{array}$ & $\begin{array}{c}\text { Lowest } \\
\text { of } 6 \text { oven } \\
\text { ther- } \\
\text { mome- } \\
\text { ters }\end{array}$ & $\begin{array}{c}\text { Ther- } \\
\text { mometer } \\
\text { in brick } \\
101\end{array}$ & $\begin{array}{l}\text { Change of } \\
\text { reading of } \\
\text { gage } H \text { on } \\
\text { brick } 101\end{array}$ & $\begin{array}{c}\text { Coefficient } \\
\text { of thermal } \\
\text { expansion } \\
\text { of brick } \\
101\end{array}$ & $\begin{array}{l}\text { Change of } \\
\text { reading of } \\
\text { gage } F \text { on } \\
\text { brick } 122\end{array}$ & $\begin{array}{l}\text { Coefficient } \\
\text { of thermal } \\
\text { expansion } \\
\text { of brick } 122\end{array}$ \\
\hline 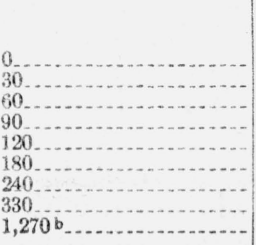 & $\begin{array}{l}{ }^{\circ} C \\
-10.9 \\
+3.7 \\
25.0 \\
41.0 \\
42.3 \\
42.4 \\
42.2 \\
41.8 \\
38.5\end{array}$ & $\begin{array}{l}{ }^{\circ} C \\
-10.9 \\
+2.4 \\
20.9 \\
36.2 \\
39.2 \\
41.1 \\
41.5 \\
41.6 \\
38.4\end{array}$ & $\begin{array}{l}{ }^{\circ} C \\
-10.9 \\
-8.0 \\
+1.7 \\
14.7 \\
26.5 \\
36.2 \\
39.7 \\
41.0 \\
38.5\end{array}$ & $\begin{array}{r}\text { Millionths } \\
\text { in. per in. } \\
0 \\
68 \\
210 \\
317 \\
332 \\
337 \\
339 \\
341 \\
321\end{array}$ & $\begin{array}{l}\text { Millionths } \\
\text { per }{ }^{\circ} \mathrm{C}\end{array}$ & $\begin{array}{r}\text { Millionths } \\
\text { in. per in. } \\
0 \\
75 \\
230 \\
353 \\
378 \\
387 \\
390 \\
392 \\
368\end{array}$ & $\begin{array}{r}\text { Millionths } \\
\text { per }{ }^{\circ} C\end{array}$ \\
\hline
\end{tabular}

srom $-10.9^{\circ} \mathrm{C}$ to the temperature given in the third column.

b Heating current reduced slightly and oven allowed to stand all night for this reading.

The surprisingly small error caused by the temperature of the bricks, gages, and oven not being equal may be accounted for by noting in table 4 that the thermal expansion of the gages was about twice that of the bricks and that they were mounted on the surface of the bricks where their temperature would be about midway between that of the bricks and that of the oven thermometers. Thus, if the temperature of the oven is $40^{\circ} \mathrm{C}$, of the gage $35^{\circ} \mathrm{C}$, and of the brick $30^{\circ} \mathrm{C}$, and the coefficient for the gage is 12 millionths per ${ }^{\circ} \mathrm{C}$ and for 
the brick 6 millionths per ${ }^{\circ} \mathrm{C}$, both would be 0.000060 inch per inch shorter than they would be if all temperatures were $40^{\circ} \mathrm{C}$. There would then be no relative change in length, and the gage would read the same as it would if all the temperatures were $40^{\circ} \mathrm{C}$. Several tests were made on other bricks and with the temperature going down as well as up, with similar results. This automatic compensation was not used to shorten the time required for the tests and would be less effective for bricks for which the thermal expansion was substantially different from those of the bricks given in table 3 . For most of the bricks it lessens the likelihood of small differences between the temperatures of the bricks and gages, causing errors in the thermal expansions obtained.

TABLE 4.-Constanis $a$ and $b$ for expressing the thermal expansion as $a$ two-term equation in powers of the lemperature

\begin{tabular}{|c|c|c|c|c|c|c|c|}
\hline $\begin{array}{c}\text { Number of } \\
\text { brick }\end{array}$ & $\begin{array}{c}\text { Constant } \\
a\end{array}$ & $\begin{array}{c}\text { Constant } \\
b\end{array}$ & $\begin{array}{c}\text { Thermal } \\
\text { expansion, } \\
-10^{\circ} \text { to } \\
+40^{\circ} \mathrm{C}\end{array}$ & $\begin{array}{c}\text { Number of } \\
\text { brick }\end{array}$ & $\begin{array}{c}\text { Constant } \\
a\end{array}$ & $\begin{array}{c}\text { Constant } \\
b\end{array}$ & $\begin{array}{l}\text { Therinal } \\
\text { expansion, } \\
-10^{\circ} \text { to } \\
+40^{\circ} \mathrm{C}\end{array}$ \\
\hline $\begin{array}{l}53 \\
54 \\
64 \\
69 \\
70\end{array}$ & $\begin{array}{r}\times 10^{-8} \\
5.05 \\
4.95 \\
5.20 \\
5.65 \\
5.40\end{array}$ & $\begin{array}{c}\times 10^{-6} \\
0.017 \\
.018 \\
.013 \\
.012 \\
.012\end{array}$ & $\begin{array}{l}\text { Millionths } \\
\text { per }{ }^{\circ} \mathrm{C} \\
5.55 \\
5.50 \\
5.60 \\
6.00 \\
5.75\end{array}$ & $\begin{array}{l}100 \\
104 \\
105 \\
113 \\
114\end{array}$ & $\begin{array}{r}\times 10^{-6} \\
4.80 \\
5.55 \\
5.50 \\
3.55 \\
3.90\end{array}$ & $\begin{array}{r}\times 10^{-9} \\
.012 \\
.012 \\
.012 \\
.011 \\
.010\end{array}$ & $\begin{array}{l}\text { Millionths } \\
\text { per }{ }^{\circ} \mathrm{C} \\
5.15 \\
5.80 \\
5.85 \\
3.90 \\
4.20\end{array}$ \\
\hline $\begin{array}{l}76 \\
81 \\
82 \\
85 \\
86 \ldots \ldots\end{array}$ & $\begin{array}{l}4.85 \\
4.95 \\
4.90 \\
4.65 \\
4.75\end{array}$ & $\begin{array}{l}.011 \\
.012 \\
.012 \\
.013 \\
.012\end{array}$ & $\begin{array}{l}5.20 \\
5.30 \\
5.25 \\
5.05 \\
5.10\end{array}$ & $\begin{array}{l}115 \\
133 \\
134 \\
135 \\
136\end{array}$ & $\begin{array}{l}3.95 \\
5.95 \\
6.15 \\
5.85 \\
4.95\end{array}$ & $\begin{array}{l}.012 \\
.013 \\
.013 \\
.011 \\
.012\end{array}$ & $\begin{array}{l}4.30 \\
6.35 \\
6.55 \\
6.20 \\
5.30\end{array}$ \\
\hline $\begin{array}{l}89 \\
90 \\
92 \\
94 \\
95\end{array}$ & $\begin{array}{l}4.85 \\
4.45 \\
2.90 \\
8.10 \\
5.56\end{array}$ & $\begin{array}{l}.013 \\
.015 \\
.013 \\
.009 \\
.013\end{array}$ & $\begin{array}{l}5.25 \\
4.90 \\
3.30 \\
8.35 \\
5.90\end{array}$ & $\begin{array}{l}137 \\
138 \\
\text { Standard bar... } \\
\text { Gage } H \text {. } \\
\text { Gage } F . . .\end{array}$ & $\begin{array}{r}11.55 \\
10.85 \\
9.40 \\
11.14 \\
11.32 \\
11.42\end{array}$ & $\begin{array}{l}.029 \\
.028 \\
.028 \\
.0132 \\
.0153 \\
.0148\end{array}$ & $\begin{array}{l}12.40 \\
11.70 \\
10.20 \\
11.54 \\
11.78 \\
11.80\end{array}$ \\
\hline
\end{tabular}

In some of the tests the expansion measured as the temperature increased was substantially different from that as the temperature decreased, as shown in table 5. This might be taken to indicate an irreversible change in the length of the brick due to a change in water content or to the temperature changes. However, as other tests on the same brick frequently did not show this change in length, it probably was due to a slight shifting or creep of the plates on which the gages were mounted rather than to changes in length of the brick. The average of the values from such tests was usually in good agreement with others which did not show this apparent change in length.

The differences between the thermal expansions of the gages, bricks, and calibrated bars, for the small temperature range used in this investigation, are very nearly proportional to the temperature. Since the differential method was used, the variation of the temperature range of several degrees does not affect appreciably the values of the thermal expansions obtained. 
TABre 5.-Thermal expansion data, $-10^{\circ}$ to $+40^{\circ} \mathrm{C}$, for two bricks, with 6-inch gages

\begin{tabular}{|c|c|c|c|c|c|}
\hline $\begin{array}{l}\text { Brick number } \\
\text { Gage }\end{array}$ & $\begin{array}{l}121 \\
H\end{array}$ & $\stackrel{121}{F}$ & $\stackrel{122}{\not}$ & $\stackrel{122}{H}$ & $\frac{122}{F}$ \\
\hline & \multicolumn{5}{|c|}{$\begin{array}{c}\text { Change in reading of gage, millionths inch por inch } \\
\text { per }{ }^{\circ} \mathrm{C}\end{array}$} \\
\hline $\begin{array}{l}\text { Temperature decreasing } \\
\text { Temperature increasing } \\
\text { Temperature decreasing } \\
\text { Temperature increasing }\end{array}$ & $\begin{array}{l}7.41 \\
7.19 \\
7.39 \\
7.30\end{array}$ & $\begin{array}{l}\text { 7. } 38 \\
7.39 \\
7.41 \\
7.38\end{array}$ & $\begin{array}{l}7.63 \\
7.49 \\
7.54 \\
7.49\end{array}$ & $\begin{array}{l}7.53 \\
7.50 \\
7.52 \\
7.52\end{array}$ & $\begin{array}{l}7.58 \\
7.47 \\
7.54 \\
7.48\end{array}$ \\
\hline Average & 7.32 & 7.39 & 7.51 & 7.52 & 7.52 \\
\hline $\begin{array}{l}\text { Coeffcient of thermal expansion of gage } \\
\text { Coefficlent of thermal expansion of brick } \\
\text { Final average } \\
\text { Standard deviation }\end{array}$ & 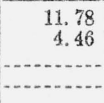 & $\begin{array}{r}11.86 \\
4.47 \\
4.47 \\
0.01\end{array}$ & 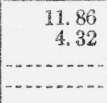 & $\begin{array}{r}11.78 \\
4.26\end{array}$ & $\begin{array}{r}11.86 \\
4.34 \\
4.31 \\
0.04\end{array}$ \\
\hline
\end{tabular}

There is some evidence in the data that the 2-inch gages gave a value of the coefficient of expansion about 0.2 millionths per ${ }^{\circ} \mathrm{C}$ higher than that given by the 6 -inch gages. Tests were made with both gages on similar bricks, 1 to 5,6 to 11 , and 12 to 18 , and both gages were used on the same bricks for the seven given at the end of table 6 .

The values of the coefficient of thermal expansion found by means of the 2-inch gages for both halves of the same brick agreed within 0.2 millionth per ${ }^{\circ} \mathrm{C}$, except for bricks 12 and 15 , which are unusually soft bricks. Of course, there is no reason why the thermal expansions of both halves of a brick should be the same, but the fact that, except for this one type, the independently determined expansions of the two halves agreed so closely leads one to expect that the random error in the values is less than this amount. A systematic error would not be disclosed by this comparison. 
TABLE 6.-Coefficient of thermal expansion of 189 bricks over the temperature range $-10^{\circ}$ to $+40^{\circ} \mathrm{C}$. with description and the data for the other physical tests made on the same bricks

[C, clay; S, shale; F, fire-clay; SL, sand-lime; SM, solt mud; SC, side cut; DP, dry press; EC, end cut; DD, down draft; UD, up draft]

\begin{tabular}{|c|c|c|c|c|c|c|c|c|c|c|c|c|c|c|}
\hline $\begin{array}{l}\text { Brick } \\
\text { No.a }\end{array}$ & $\begin{array}{l}\text { Mate- } \\
\text { rial }\end{array}$ & $\begin{array}{l}\text { Method of } \\
\text { forming }\end{array}$ & Kiln & $\begin{array}{l}\text { Manufacturer's } \\
\text { grade name }\end{array}$ & Locality of production & $\begin{array}{l}\text { Com- } \\
\text { pressive } \\
\text { strength }\end{array}$ & $\begin{array}{c}\text { Modulus } \\
\text { of rup- } \\
\text { ture }\end{array}$ & $\begin{array}{l}\text { 5-hour } \\
\text { boiling } \\
\text { absorp- } \\
\text { tion, } B\end{array}$ & $\begin{array}{c}\text { 24-hour } \\
\text { water ab- } \\
\text { sorption, } \\
C\end{array}$ & $\begin{array}{l}\text { Satura- } \\
\text { tion co- } \\
\text { efficient } \\
(C / B)\end{array}$ & $\begin{array}{l}\text { Coefficient } \\
\text { of thermal } \\
\text { expansion }\end{array}$ & $\begin{array}{l}\text { Num- } \\
\text { ber of } \\
\text { tests }\end{array}$ & $\begin{array}{c}\text { Root mean } \\
\text { square of } \\
\text { deviation } \\
\text { from mean }\end{array}$ & $\begin{array}{l}\text { Brick } \\
\text { No. }\end{array}$ \\
\hline $\begin{array}{l}1 \mathrm{a} \ldots \ldots \\
1 \mathrm{~b} \ldots \ldots \\
2 \mathrm{a} \ldots \ldots \\
2 \mathrm{~b} \ldots \ldots \\
3 \mathrm{a} \ldots \ldots \\
4 \ldots \ldots \\
5 \ldots \ldots\end{array}$ & $\begin{array}{l}\mathrm{F} \\
\mathrm{F} \\
\mathrm{F} \\
\mathrm{F} \\
\mathrm{F} \\
\mathrm{F} \\
\mathrm{F} \\
\mathrm{F}\end{array}$ & $\begin{array}{l}\mathrm{SC} \\
\mathrm{SC}- \\
\mathrm{SC}- \\
\mathrm{SC}- \\
\mathrm{SC}- \\
\mathrm{SC}- \\
\mathrm{SC}- \\
\mathrm{SO}\end{array}$ & & Kittanning_.. & $\begin{array}{c}\begin{array}{c}\text { Western Pennsylvania } \\
\text { do }\end{array} \\
\text { do do } \\
\text { do do }\end{array}$ & \begin{tabular}{|c}
$\mathrm{Lb} / \mathrm{in} .^{2}$ \\
12,500 \\
15,900 \\
14,200 \\
20,600 \\
18,200
\end{tabular} & $\begin{array}{r}\text { Lb/in. }{ }^{2} \\
3,100 \\
3,100 \\
3,500 \\
2,500 \\
2,600\end{array}$ & \begin{tabular}{|r|} 
Percent \\
dry weight \\
1.8 \\
1.5 \\
1.3 \\
1.2 \\
1.5 \\
1.4 \\
1.8 \\
1.4
\end{tabular} & $\begin{array}{r}\text { Percent } \\
\text { dry weight } \\
0.7 \\
.6 \\
.3 \\
.3 \\
.3 \\
.2 \\
.4 \\
.3\end{array}$ & - & $\begin{array}{r}\text { Millionths } \\
\text { per }{ }^{\circ} \mathrm{C} . \\
\\
* 3.2 \\
\\
* 3.1 \\
\\
* 3.3 \\
* 3.2 \\
* 3.3 \\
* 3.4 \\
3.05 \\
3.05\end{array}$ & \begin{tabular}{l|l}
5 & \\
4 & \\
8 & \\
9 & \\
7 & \\
8 & \\
3 & \\
1 &
\end{tabular} & $\begin{array}{r}\text { Millionths } \\
\text { per }{ }^{\circ} \mathrm{C} \\
0.08 \\
0.05 \\
.08 \\
.03 \\
.06 \\
.05 \\
.05\end{array}$ & $\begin{array}{r}1 \mathrm{a} \\
1 \mathrm{~b} \\
2 \mathrm{a} \\
2 \mathrm{~b} \\
3 \mathrm{a} \\
3 \mathrm{~b} \\
4 \\
5\end{array}$ \\
\hline Avg - & $\cdots$ & & & & & 16,300 & 3,000 & 1.5 & .4 & ....... & $* 3.1$ & ‥- & - & A $\nabla g$. \\
\hline $\begin{array}{l}6 \mathrm{a} \ldots \ldots \\
6 \mathrm{~b} \ldots \ldots \\
7 \mathrm{a} \ldots \ldots \\
7 \mathrm{~b} \ldots \ldots \ldots \\
8 \mathrm{a} \ldots \ldots \ldots \\
9 \mathrm{~b} \ldots \ldots . . \\
9 \mathrm{~b} \ldots \ldots \\
10 \ldots \ldots \\
11\end{array}$ & $\begin{array}{l}\mathrm{S} \\
\mathrm{S} \\
\mathrm{S} \\
\mathrm{S} \\
\mathrm{S} \\
\mathrm{S} \\
\mathrm{S} \\
\mathrm{S} \\
\mathrm{S} \\
\mathrm{S}\end{array}$ & $\begin{array}{l}\text { SC } \\
\text { SC } \\
\text { SC } \\
\text { SC } \\
\text { SC } \\
\text { SC } \\
\text { SC } \\
\text { SC } \\
\text { SC } \\
\text { SC }\end{array}$ & & & 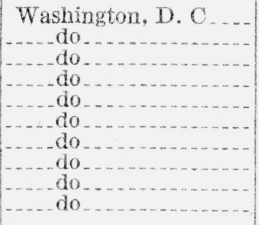 & $\begin{array}{l}4,400 \\
6,200\end{array}$ & $\begin{array}{l}1,180 \\
1,340 \\
1,550 \\
1,020 \\
1,370\end{array}$ & $\begin{array}{r}10.7 \\
10.7 \\
12.1 \\
12.5 \\
10.2 \\
9.3 \\
11.8 \\
10.8 \\
12.5 \\
12.3\end{array}$ & $\begin{array}{l}6.5 \\
7.4 \\
8.9 \\
9.4 \\
6.8 \\
5.1 \\
7.6 \\
7.9 \\
9.7 \\
9.3\end{array}$ & $\begin{array}{l}0.64 \\
.69 \\
.73 \\
.75 \\
.67 \\
.55 \\
.64 \\
.73 \\
.78 \\
.76\end{array}$ & $\begin{array}{l}* 6.5 \\
* 6.3 \\
* 6.1 \\
* 6.2 \\
* 6.3 \\
* 6.1 \\
* 6.3 \\
* 6.3 \\
5.85 \\
6.00\end{array}$ & $\begin{array}{l}3 \\
4 \\
2 \\
3 \\
4 \\
6 \\
7 \\
3 \\
3 \\
1\end{array}$ & $\begin{array}{l}0.04 \\
.04 \\
.05 \\
.11 \\
.08 \\
.06 \\
.05 \\
.05 \\
.07 \\
. . .\end{array}$ & $\begin{array}{l}6 \mathrm{a} \\
6 \mathrm{~b} \\
7 \mathrm{a} \\
7 \mathrm{~b} \\
8 \mathrm{a} \\
8 \mathrm{~b} \\
9 \mathrm{a} \\
9 \mathrm{~b} \\
10 \\
11\end{array}$ \\
\hline $\mathrm{Avg}_{-}$ & & & & & & 5,400 & 1,300 & 11.3 & 8.3 & .73 & $* 6.1$ & & n......... & Avg. \\
\hline $\begin{array}{l}12 \mathrm{a} \ldots \ldots \\
12 \mathrm{~b} \ldots \ldots \\
13 \mathrm{a} \ldots \ldots \\
13 \mathrm{~b} \ldots \ldots \\
14 \mathrm{a} \ldots \ldots \\
14 \mathrm{~b} \ldots \ldots \\
15 \mathrm{a} \ldots \ldots \\
16 \mathrm{~b} \ldots \ldots \\
17 \ldots \ldots \ldots \\
18 \ldots \ldots\end{array}$ & $\begin{array}{l}\mathrm{C} \\
\mathrm{C} \\
\mathrm{C} \\
\mathrm{C} \\
\mathrm{C} \\
\mathrm{C} \\
\mathrm{C} \\
\mathrm{C} \\
\mathrm{C} \\
\mathrm{C} \\
\mathrm{C}\end{array}$ & 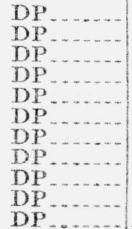 & & & 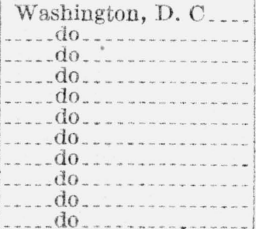 & $\begin{array}{l}2,150 \\
2,070 \\
2,080\end{array}$ & $\begin{array}{l}130 \\
170 \\
200\end{array}$ & $\begin{array}{l}19.8 \\
20.2 \\
19.5 \\
19.1 \\
17.8 \\
19.7 \\
14.5 \\
14.2 \\
14.4 \\
18.1 \\
17.6\end{array}$ & $\begin{array}{l}17.2 \\
17.5 \\
11.6 \\
16.8 \\
14.3 \\
15.7 \\
11.2 \\
10.9 \\
12.0 \\
15.4 \\
14.8\end{array}$ & $\begin{array}{l}.87 \\
.87 \\
.60 \\
.88 \\
.80 \\
.80 \\
.77 \\
.67 \\
.83 \\
.85 \\
.84\end{array}$ & $\begin{array}{l}* 7.5 \\
* 6.5 \\
* 6.7 \\
* 6.6 \\
* 7.2 \\
* 7.0 \\
* 6.9 \\
* 6.1 \\
6.45 \\
6.85 \\
6.50\end{array}$ & $\begin{array}{l}8 \\
4 \\
5 \\
7 \\
5 \\
6 \\
6 \\
7 \\
2 \\
2 \\
2\end{array}$ & $\begin{array}{r}0.18 \\
.21 \\
.06 \\
.09 \\
.08 \\
.06 \\
.13 \\
.06 \\
.03 \\
.01 \\
.04\end{array}$ & $\begin{array}{r}12 \mathrm{a} \\
12 \mathrm{~b} \\
13 \mathrm{a} \\
13 \mathrm{~b} \\
14 \mathrm{a} \\
14 \mathrm{~b} \\
15 \mathrm{a} \\
15 \mathrm{~b} \\
16 \\
17 \\
18\end{array}$ \\
\hline Avg .. & & & & & 6. & 2,600 & 300 & 17.5 & 14.9 & .85 & *6.7 & & & Avg. \\
\hline
\end{tabular}




\begin{tabular}{|c|c|c|c|c|c|c|c|c|c|c|c|c|c|}
\hline \multirow{2}{*}{$\begin{array}{l}19 \ldots \ldots \\
200 \ldots \ldots\end{array}$} & C & $\mathrm{SC} \ldots \ldots$ & DD ..... & Select White..... & Whitewater, Wis .... & 1,900 & 400 & 31.1 & 25.6 & 0.82 & $* 8.5$ & 6 & 0.16 \\
\hline & c & $8 M$ & Gcove... & Stock & Detroit, Mich & 4,400 & 590 & 22.7 & 18.8 & .83 & 76.0 & 4 & .07 \\
\hline & $\mathrm{C}$ & SM. & UD..... & Select Bard. & Kingston, N. $\mathrm{Y}$. & 6,100 & 780 & 19.0 & 14. 9 & .78 & ${ }^{*} 6.2$ & 4 & .16 \\
\hline $22 \ldots$ & $\mathrm{C}$ & $\mathrm{SM}$. & Scove... & Kiln run..... & Lebanon, N. H. & 8,000 & 1,950 & 7.1 & 2. 9 & .41 & $* 5.2$ & 5 & .03 \\
\hline 23 . & 5 & $D P$. & UD .... & ..... do..... & D'Hamis, Tex.. & 6,800 & 980 & 20.8 & 18.7 & .90 & $* 6.0$ & 6 & .07 \\
\hline 24 & 8 & $\mathrm{DP}$. & UD... & Arch & Pueblo, Colo... & 5,500 & 1,060 & 15.3 & 12.7 & .83 & $* 6.6$ & 6 & .05 \\
\hline 25 . & C\&s & DP. & Scove. & Picked Reds & Sheridan, Wyo.. & 4,200 & 320 & 13.8 & 11.1 & .80 & $\# 6.5$ & 4 & .09 \\
\hline 26 & $\mathrm{~s}$ & SC. & UD & Medium & Pueblo, Colo & 7,200 & 1,370 & 14. 7 & 12.6 & .86 & $* 6.7$ & 4 & .08 \\
\hline 27 & C & $\mathrm{EC}$. & Dutch... & Common hard... & Sharon Hill, $\mathrm{Pa}$.. & 7,400 & 780 & 12.1 & 7.6 & .63 & $* 6.2$ & 4 & 10 \\
\hline 28 & $s$ & BO. & Tunuel & Common. & Cleveland, Ohio. & 6,700 & 700 & 11.8 & 9.3 & .79 & $* 6.5$ & 6 & .07 \\
\hline 29. & $\mathrm{C}$ & 80 & & Clinker... & Richmond, Va... & 7,000 & 1,350 & 11.8 & 7.5 & .66 & $* 5.9$ & 5 & .12 \\
\hline 30 & $\mathrm{~s}$ & DP. & $\mathrm{DD} \ldots$ & Red Face_- & Birmingham, Ala & 15,800 & 2,160 & 7.0 & 4.1 & .59 & 4.7 & 4 & 11 \\
\hline 31. & $\mathrm{C}$ & SMT. & Scove. & Exterior - & Detroit, Mich & 8,600 & 2,320 & 10.8 & 4.4 & .41 & $* 5.7$ & 5 & .08 \\
\hline 32 & $\mathrm{~s}$ & $\mathrm{SC}$. & DD .... & Hard ...... & Alton, 11 . & 16,700 & 2,370 & 3.0 & .7 & .23 & ${ }^{*} 6.0$ & 5 & 08 \\
\hline $33 \ldots$ & $\mathrm{F}$ & $\mathrm{SC}$ & DD $\ldots$ & Common & Alliance, Ohio & 21,000 & 2,500 & 5.1 & 1.3 & .25 & & 4 & 06 \\
\hline 34 & $\mathrm{C}$ & $\mathrm{SC}$ & Dutch. & Black Arch. & Baltimore, Md. & 13,000 & 2,100 & 4.2 & 1.4 & .33 & $* 6.0$ & 4 & .14 \\
\hline 35. & $\mathrm{C}$ & $S M$ & Scove.. & Kiln run .... & Lebanon, N. H. & 14,600 & 2,500 & 8.6 & 5.5 & .64 & $* 5.3$ & 5 & .07 \\
\hline 36. & s & $\mathrm{SO}_{-}$ & DD .... & Clinker... & Allentown, $\mathrm{Pa}$ & 12,000 & 2,060 & 5.2 & 2.6 & .50 & $* 6.8$ & 6 & .16 \\
\hline 37 & $\mathrm{C}$ & $\mathrm{SC}-1$ & DD... & Reds $\ldots . .$. & Evansville, Ind. & 14,100 & 2,500 & 3.2 & .5 & .15 & $* 5.9$ & 3 & .04 \\
\hline $38 \ldots$ & $\mathrm{C}$ & SM. & Scove.. & Clinker... & Cleveland, Ohio .. & 10,100 & 1,700 & 1.5 & .5 & & $* 5.3$ & 3 & 11 \\
\hline 39 & C & SMI & DD & $\ldots$ do $\ldots . . . . . .$. & New London, W is & 9,500 & 1,130 & 15.5 & 10.7 & .69 & $*_{6.7} .7$ & 3 & 05 \\
\hline $40 \ldots$ & $\mathrm{C}$ & $\mathrm{SM}$. & DD. & Semiantique. & Upper Sandusky, Ohio. & 16,000 & 3,200 & .8 & .2 & & ${ }^{*} 6$. & 4 & .03 \\
\hline 41a.. & $\mathrm{C}$ & SM. & & & & & & 20.0 & 15.4 & .77 & *5.7 & 3 & .07 \\
\hline $41 \mathrm{~b}$ & c & SM & & & & 3.700 & 430 & 19.3 & 14.3 & .74 & $* 5.7$ & 3 & .05 \\
\hline $42 \mathrm{a}$ & C & $\mathrm{SM}_{\mathrm{SM}}$ & & & & & & 18. 2 & 13.7 & .75 & $\begin{array}{l}* 5.9 \\
* 5.8\end{array}$ & 3 & .12 \\
\hline $42 \mathrm{~b}$ & $\mathrm{C}$ & & & & & 4,000 & 890 & & & & & 7 & \\
\hline $\operatorname{avg} e_{-}$ & $\cdots$ & ..... & & & & 3,800 & 660 & 18.8 & 14.2 & .75 & *5. 8 & & \\
\hline 43a.. & $\mathrm{s}$ & $\mathrm{SC}$ & & Sewer brick & & & & 4.5 & 2.4 & .53 & $* 6.5$ & 9 & .06 \\
\hline $43 \mathrm{~b}$. & $\mathrm{s}$ & SC. & ...... & do & & 13,300 & 3,100 & 4.6 & 2.6 & .56 & $* 6.4$ & 3 & .11 \\
\hline avg . & ....... & ...... & …..... & -......... & .... $>$ & $\ldots . .$. & & 4.6 & 2.5 & .54 & $* 6.4$ & & \\
\hline $44 a \ldots$ & $\mathrm{s}$ & $\mathrm{SC}$ & & Sewer brick. & & & & 6.1 & 3.8 & .62 & $* 6.6$ & 5 & .05 \\
\hline $44 \mathrm{~b}$. & $\mathrm{s}$ & $\mathrm{SC}-$ & ...... & ..... do do......... & & 8,100 & 1,950 & 6.0 & 3.8 & .63 & *6. 6 & 3 & .10 \\
\hline avg... & $\ldots$ & - & (............ & (.). & $\ldots . . .$. & & & 6.0 & 3.8 & .62 & ${ }^{*} 6.6$ & & \\
\hline $45 a \ldots \ldots$ & s & $\mathrm{SC}$ & $\ldots$ & Sewer brick & & & & 5.5 & 3.5 & .64 & $* 6.5$ & 3 & .06 \\
\hline $45 \mathrm{~b} \ldots \ldots$ & $\mathrm{s}$ & $\mathrm{SC}$ & ........... & ..... do & & 10,900 & 3,300 & 5.2 & 3.3 & .63 & $* 6.7$ & 4 & .07 \\
\hline avg ... & ..... & & (n) & - & - & .......... & .......... & 5.4 & 3.4 & .63 & $* 6.6$ & ... & \\
\hline 468. & $\mathrm{C}$ & Semi-DP.. & $\ldots$ & $\ldots . .$. & Washington, D. C & & & & 10.1 & & $* 6.9$ & 2 & \\
\hline $46 \mathrm{~b}$ & C & .. do do........ & & & 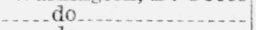 & 4,900 & 810 & 12.5 & 10.1 & .81 & $* 6.7$ & 2 & .08 \\
\hline $47 \mathrm{a}$ & C & ... do & & & ...do... & & & 11.6 & 8.9 & .77 & ${ }^{7} 6.6$ & 3 & .13 \\
\hline $47 \mathrm{~b} \ldots .$. & $\mathrm{C}$ & .... do & & & ..... do... & 5,300 & 1,000 & 11.8 & 9.3 & .79 & ${ }^{*} 6.5$ & 3 & .04 \\
\hline $\operatorname{avg}{ }_{-}$ & ..... & & ....... & ...... & & 5,100 & 900 & 12.1 & 9.6 & .79 & $* 6.7$ & & \\
\hline
\end{tabular}

See footnotes at end of table. 
TABLE 6.-Coefficient of thermal expansion of 139 bricks over the temperature range -, $0^{\circ}$ to $+40^{\circ}$, with description and the data for the other physical tests made on the same bricks-Continued

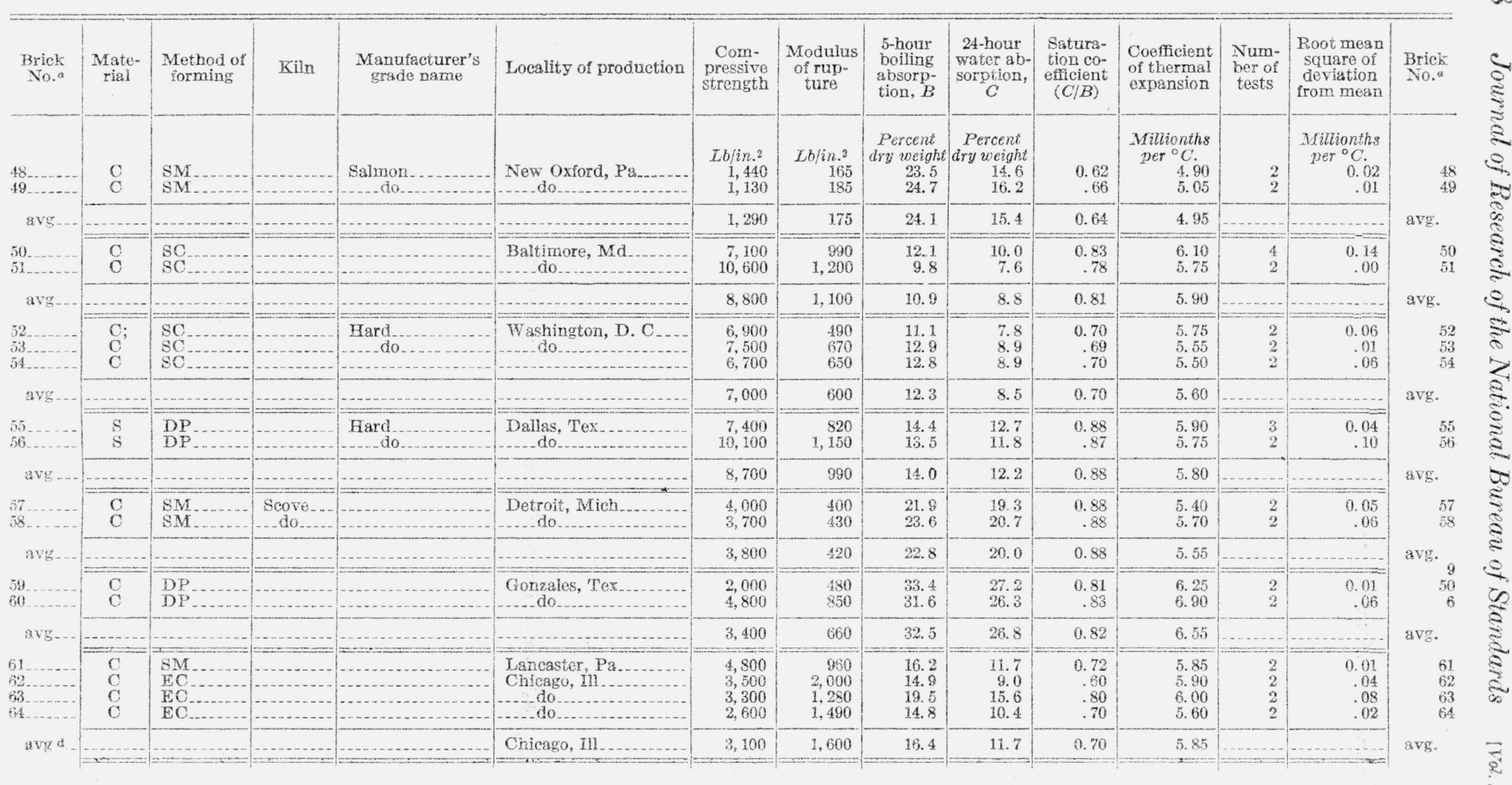




\begin{tabular}{|c|c|c|c|c|c|c|c|c|c|c|c|c|c|}
\hline $\begin{array}{l}65 \\
66\end{array}$ & $\stackrel{\mathrm{C}}{\mathrm{C}}$ & $\begin{array}{l}\mathrm{SM} \\
\mathrm{SM}\end{array}$ & -..... & (n) & Hudson Valley, N. Y & $\begin{array}{l}2,700 \\
6,200\end{array}$ & $\begin{array}{l}380 \\
400\end{array}$ & $\begin{array}{l}23.7 \\
19.3\end{array}$ & $\begin{array}{l}19.7 \\
13.7\end{array}$ & $\begin{array}{r}0.83 \\
.71\end{array}$ & $\begin{array}{l}6.55 \\
5.75\end{array}$ & $\frac{2}{2}$ & $\begin{array}{r}0.01 \\
.04\end{array}$ \\
\hline avg.. & -......... & ........ & & & (-n........ & 4,400 & 390 & 21.5 & 16.7 & 0.77 & 6.15 & & \\
\hline $\begin{array}{l}67 \\
68\end{array}$ & $\begin{array}{l}\mathrm{C} \\
\mathrm{C}\end{array}$ & $\begin{array}{l}\mathrm{SM} \\
\mathrm{SM}\end{array}$ & & & $\begin{array}{l}\text { Hudson Valley, N. Y } \\
\text { do }\end{array}$ & $\begin{array}{l}2,840 \\
8,400\end{array}$ & $\begin{array}{l}320 \\
970\end{array}$ & $\begin{array}{l}24.3 \\
14.5\end{array}$ & $\begin{array}{l}22.2 \\
11.8\end{array}$ & $\begin{array}{r}0.91 \\
.81\end{array}$ & $\begin{array}{l}5.95 \\
5.75\end{array}$ & $\begin{array}{l}3 \\
2\end{array}$ & $\begin{array}{r}0.04 \\
.05\end{array}$ \\
\hline avg... & 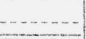 & ........ & & & ………… & 5,600 & 650 & 19.4 & 17.0 & 0.86 & 5.85 & ... & ....... \\
\hline $\begin{array}{l}69 \\
70 \\
71 \ldots \ldots\end{array}$ & $\begin{array}{l}\mathrm{S} \\
\mathrm{S} \\
\mathrm{S}\end{array}$ & $\begin{array}{l}\mathrm{SC} \\
\mathrm{SC} \\
\mathrm{SC}\end{array}$ & & & $\begin{array}{l}\text { Martinsburg, W. Va. } \\
\text { do }\end{array}$ & $\begin{array}{l}13,200 \\
16,000 \\
13,700\end{array}$ & $\begin{array}{r}540 \\
1,650 \\
1,910\end{array}$ & $\begin{array}{l}5.2 \\
2.6 \\
5.3\end{array}$ & $\begin{array}{l}2.9 \\
0.9 \\
2.9\end{array}$ & $\begin{array}{l}0.56 \\
.35 \\
.55\end{array}$ & $\begin{array}{l}6.00 \\
5.75 \\
6.05\end{array}$ & $\begin{array}{l}2 \\
2 \\
2\end{array}$ & $\begin{array}{r}0.02 \\
.00 \\
.01\end{array}$ \\
\hline avg.- & $\cdots$ & ........ & $\ldots$ & - & - & 14,300 & 1,370 & 4.4 & 2.2 & 0.49 & 5.95 & ... & ......... \\
\hline 72 & $\begin{array}{l}\mathrm{S} \\
\mathrm{S}\end{array}$ & $\begin{array}{l}\mathrm{SC}_{-} \\
\mathrm{SC}\end{array}$ & & - & Martinsburg, W. Va. & $\begin{array}{l}6,500 \\
6,000\end{array}$ & $\begin{array}{r}1,130 \\
730\end{array}$ & $\begin{array}{l}12.0 \\
13.1\end{array}$ & $\begin{array}{r}9.9 \\
10.9\end{array}$ & $\begin{array}{r}0.82 \\
.83 \\
\end{array}$ & $\begin{array}{l}6.85 \\
6.70\end{array}$ & $\begin{array}{l}3 \\
2 \\
\end{array}$ & $\begin{array}{r}0.07 \\
.04\end{array}$ \\
\hline avg... & 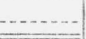 & $\ldots$ & $\ldots$ & -................. & - & 6,200 & 930 & 12.5 & 10.4 & 0.82 & 6.80 & ... & ........ \\
\hline $\begin{array}{ll}74 \\
75 \ldots \ldots\end{array}$ & $\stackrel{\mathrm{C}}{\mathrm{C}}$ & $\begin{array}{l}\text { DP.- } \\
\text { DP.- }\end{array}$ & & & Washington, D. C.... & $\begin{array}{l}2,740 \\
3,400 \\
\end{array}$ & $\begin{array}{l}280 \\
320 \\
\end{array}$ & $\begin{array}{l}17.0 \\
15.6\end{array}$ & $\begin{array}{l}14.4 \\
12.5 \\
\end{array}$ & $\begin{array}{r}0.85 \\
.80\end{array}$ & $\begin{array}{l}6.30 \\
5.65 \\
\end{array}$ & $\begin{array}{l}2 \\
2 \\
\end{array}$ & $\begin{array}{r}0.01 \\
.07\end{array}$ \\
\hline avg.- & $\cdots$ & ......... & $\ldots$ & 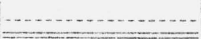 & . & 3,100 & 300 & 16. 3 & 13.4 & 0.82 & 6.00 & -.. & $\ldots$ \\
\hline $\begin{array}{l}76 \\
77 \\
78\end{array}$ & $\begin{array}{l}\mathrm{C} \\
\mathrm{C} \\
\mathrm{C}\end{array}$ & $\begin{array}{l}\text { SM } \\
\text { SM } \\
\text { SM }\end{array}$ & & $\begin{array}{l}\text { Sand struck } \\
\text { do } \\
\end{array}$ & $\begin{array}{l}\text { Lebanon, N. H } \\
\text { do } \\
\end{array}$ & $\begin{array}{r}8,600 \\
15,600 \\
11,600\end{array}$ & $\begin{array}{l}1,310 \\
1,690 \\
1,350\end{array}$ & $\begin{array}{l}9.6 \\
8.4 \\
8.4\end{array}$ & $\begin{array}{l}5.0 \\
3.7 \\
5.5 \\
\end{array}$ & $\begin{array}{l}0.52 \\
.44 \\
.65 \\
\end{array}$ & $\begin{array}{l}5.20 \\
5.15 \\
4.95\end{array}$ & $\begin{array}{l}2 \\
2 \\
2\end{array}$ & $\begin{array}{r}0.02 \\
.01 \\
.02\end{array}$ \\
\hline avg.. & -...- & .............. & $\ldots$ & $\ldots \ldots$ & - & 11,700 & 1,450 & 8.8 & 4.7 & 0.54 & 5.10 & $\ldots$ & ....... \\
\hline 79 & C & SM.... & & $\begin{array}{l}\text { Light burned, } \\
\text { sand struck. }\end{array}$ & Lebanon, N. H... & 6,300 & 910 & 17.0 & 13.9 & 0.82 & 5. 10 & 2 & 0.02 \\
\hline 80 & $\stackrel{\mathrm{C}}{\mathrm{C}}$ & $\begin{array}{l}\text { SM } \\
\text { SM }\end{array}$ & & ....do do do & - & $\begin{array}{l}3,400 \\
4,500\end{array}$ & $\begin{array}{l}460 \\
810\end{array}$ & $\begin{array}{l}19.4 \\
18.1\end{array}$ & $\begin{array}{l}16.9 \\
14.5 \\
\end{array}$ & $\begin{array}{l}.87 \\
.80\end{array}$ & $\begin{array}{l}5.45 \\
5.30\end{array}$ & $\begin{array}{l}5 \\
2 \\
\end{array}$ & $\begin{array}{l}.09 \\
.03 \\
\end{array}$ \\
\hline avg - & - n........ & $\ldots$ & & …… & - & 4,700 & 730 & 18.2 & 15.1 & 0.83 & 5.30 & -.. & 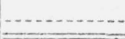 \\
\hline $\begin{array}{l}82 \\
83 \\
84 \ldots \ldots \\
85 \ldots \\
86 \ldots\end{array}$ & $\begin{array}{l}\mathrm{C} \\
\mathrm{C} \\
\mathrm{C} \\
\mathrm{C} \\
\mathrm{C}\end{array}$ & $\begin{array}{l}\text { SM } \\
\text { SM } \\
\text { SM } \\
\text { SM } \\
\text { SM }\end{array}$ & & $\begin{array}{c}\begin{array}{c}\text { Hard burned, } \\
\text { water struck. }\end{array} \\
\text { do do } \\
\text { do } \\
\end{array}$ & $\begin{array}{l}\text { Lebanon, N. H. } \\
\text { do do } \\
\text { do }\end{array}$ & $\begin{array}{l}12,700 \\
16,000 \\
13300 \\
15,400 \\
14,400\end{array}$ & $\begin{array}{l}1,130 \\
1,920 \\
2330 \\
1,140 \\
1,390 \\
\end{array}$ & $\begin{array}{l}8.3 \\
8.3 \\
6.4 \\
6.0\end{array}$ & $\begin{array}{l}2.7 \\
5.3 \\
5.2 \\
1.5 \\
1.7\end{array}$ & $\begin{array}{r}0.50 \\
.64 \\
.63 \\
.23 \\
.28\end{array}$ & $\begin{array}{l}5.25 \\
5.10 \\
5.25 \\
5.05 \\
5.10\end{array}$ & $\begin{array}{l}2 \\
2 \\
2 \\
2 \\
2\end{array}$ & $\begin{array}{l}0.05 \\
.01 \\
.05 \\
.04 \\
.02\end{array}$ \\
\hline 8vg... & - n. & - & - & & & 14,400 & 1,580 & 6.9 & 3.3 & 0.46 & 5.15 & $\ldots$ & ..... \\
\hline
\end{tabular}


TABLE 6.-Coefficient of thermal expansion of 139 bricks over the temperature range $-10^{\circ}$ to $+44^{\circ} \mathrm{C}$, with description and the data for the other physical tests made on the same bricks - Continued

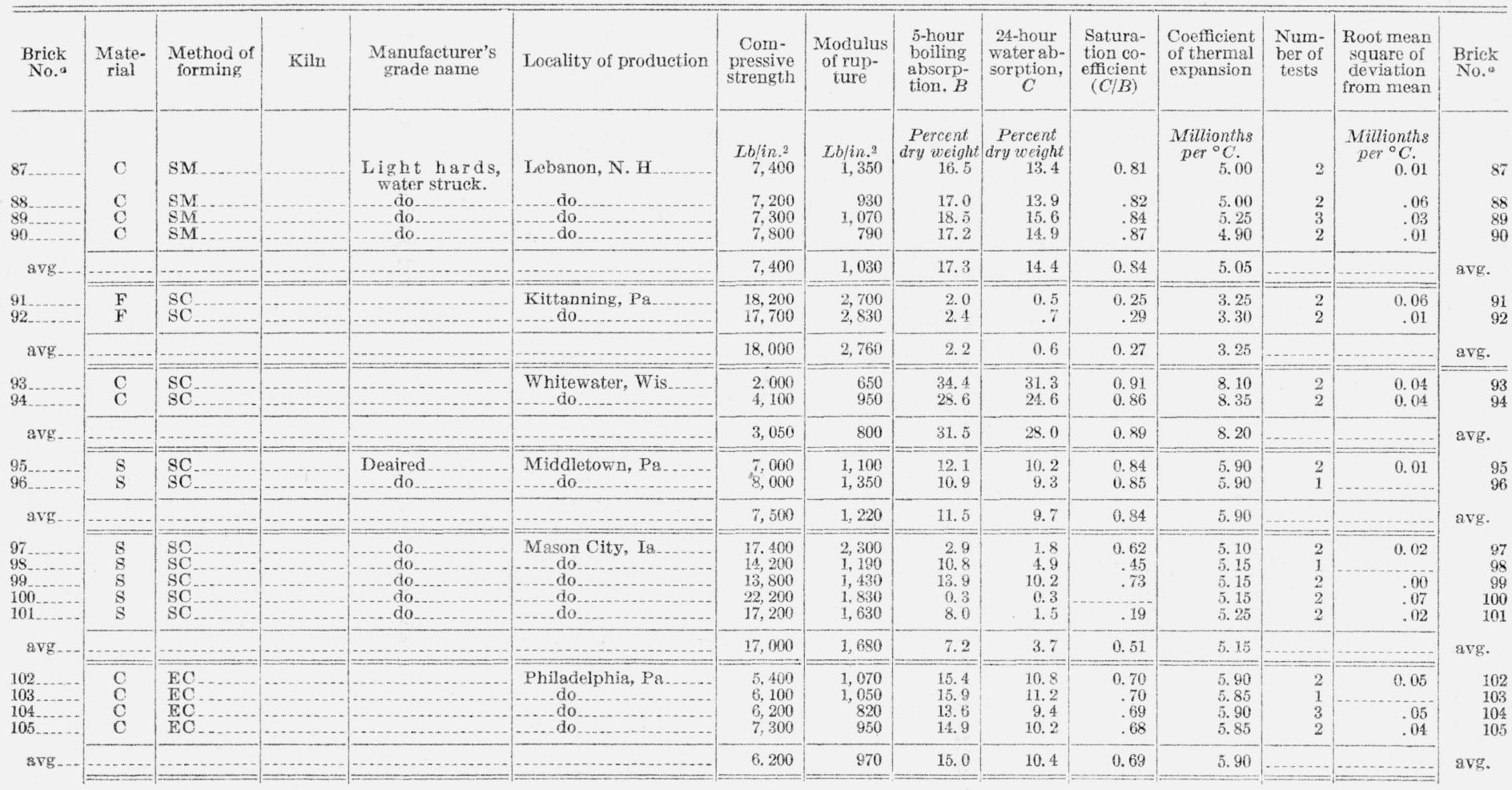




\begin{tabular}{|c|c|c|c|c|c|c|c|c|c|c|c|c|c|c|}
\hline $\begin{array}{l}106 \\
107 \\
108\end{array}$ & $\begin{array}{c}S \\
S-L \\
S-L\end{array}$ & $\mathrm{SC}$ & & Sand-lime & $\begin{array}{l}\text { St. Paul, Minn } \\
\text { Philadelphia, Pa_... }\end{array}$ & $\begin{array}{l}4,600 \\
4,100 \\
4,100\end{array}$ & $\begin{array}{l}990 \\
660 \\
500\end{array}$ & $\begin{array}{l}19.9 \\
17.0 \\
16.2\end{array}$ & $\begin{array}{l}11.8 \\
11.8 \\
12.0\end{array}$ & $\begin{array}{r}0.59 \\
.69 \\
.74\end{array}$ & $\begin{array}{r}6.30 \\
10.25 \\
10.75\end{array}$ & $\begin{array}{l}2 \\
4 \\
3\end{array}$ & $\begin{array}{r}0.03 \\
.08 \\
.03\end{array}$ & $\begin{array}{l}106 \\
107 \\
108\end{array}$ \\
\hline avge ${ }^{2}$ & $\ldots$ & …..... & -... & ............ & - & 4,100 & 580 & 16.6 & 11.9 & 0.72 & 10.50 & ......... & (n).............. & avg. \\
\hline $\begin{array}{l}109 \\
110 \\
111 \\
112 \\
113 \ldots \\
114 \\
115\end{array}$ & $\begin{array}{l}\text { F } \\
F \\
F \\
F \\
F \\
F \\
F\end{array}$ & $\begin{array}{l}\text { SC } \\
\text { SC } \\
\text { SC } \\
\text { SC } \\
\text { SC } \\
\text { SC } \\
\text { SC }\end{array}$ & 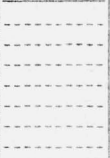 & \begin{tabular}{|l}
$\begin{array}{l}\text { Enameled } \\
\text { do }\end{array}$ \\
do \\
do do \\
do
\end{tabular} & $\begin{array}{l}\text { Plack Lick, Ohio } \\
\text { Momence, Ill } \\
\text { Columbus, Ohio } \\
\text { Canton, Ohio } \\
\text { Columbus, Ohio } \\
\end{array}$ & $\begin{array}{l}21,000 \\
11,200 \\
17,300 \\
23,600 \\
18,200 \\
19,800 \\
21,800\end{array}$ & $\begin{array}{l}1,600 \\
950 \\
1,050 \\
1,430 \\
2.230 \\
1,960 \\
2,240\end{array}$ & $\begin{array}{l}4.3 \\
8.5 \\
6.4 \\
5.6 \\
5.1 \\
4.8 \\
5.5\end{array}$ & $\begin{array}{l}0.9 \\
4.7 \\
4.2 \\
4.7 \\
2.3 \\
2.2 \\
3.1\end{array}$ & $\begin{array}{l}0.21 \\
.55 \\
.66 \\
.84 \\
.45 \\
.46 \\
.56\end{array}$ & $\begin{array}{l}\text { 4. } 05 \\
4.10 \\
4.45 \\
4.55 \\
3.90 \\
4.20 \\
4.30\end{array}$ & \begin{tabular}{l|l}
3 \\
2 \\
2 \\
2 \\
2 \\
2 \\
2 \\
2
\end{tabular} & $\begin{array}{l}0.06 \\
.01 \\
.01 \\
.00 \\
.01 \\
.03 \\
.06\end{array}$ & $\begin{array}{l}109 \\
110 \\
111 \\
112 \\
113 \\
115 \\
114\end{array}$ \\
\hline avg e & -1 & $\cdots$ & & & & 20,800 & 2,100 & 5. 2 & 2.6 & 0.51 & 4.25 & ... & ....... & avg. \\
\hline \begin{tabular}{l|}
116 \\
117 \\
118 \\
119 \\
120 \\
121 \\
122 \\
123
\end{tabular} & $\begin{array}{l}\mathrm{C} \\
\mathrm{S} \\
\mathrm{C} \\
\mathrm{S} \\
\mathrm{S} \\
\mathrm{C} \\
\mathrm{C} \\
\mathrm{C}\end{array}$ & $\begin{array}{l}\text { SC } \\
\text { SC } \\
\text { SM } \\
\text { SC } \\
\text { SC } \\
\text { SM } \\
\text { SM } \\
\text { SM }\end{array}$ & & Reclaimed & $\begin{array}{l}\text { Mason City, Iowa } \\
\text { Paducah, Kentucky } \\
\text { Cleveland, Ohio } \\
\text { do_... } \\
\text { Baltimore, Md } \\
\text { do do }\end{array}$ & $\begin{array}{r}3,800 \\
10,400 \\
7,400 \\
12,700 \\
5,500 \\
2,590 \\
2,100 \\
2,100\end{array}$ & $\begin{array}{r}760 \\
840 \\
860 \\
1.750 \\
950 \\
620 \\
620 \\
490\end{array}$ & $\begin{array}{r}25.8 \\
15.0 \\
17.4 \\
6.8 \\
12.8 \\
17.2 \\
17.0 \\
18.7\end{array}$ & $\begin{array}{r}22.2 \\
9.1 \\
14.8 \\
2.7 \\
10.4 \\
10.2 \\
9.6 \\
11.3\end{array}$ & $\begin{array}{r}0.86 \\
.61 \\
.85 \\
.40 \\
.81 \\
.59 \\
.56 \\
.60\end{array}$ & $\begin{array}{l}5.90 \\
5.35 \\
6.30 \\
5.95 \\
6.50 \\
4.45 \\
4.30 \\
4.30\end{array}$ & $\begin{array}{l}2 \\
2 \\
2 \\
2 \\
2 \\
2 \\
3 \\
2\end{array}$ & $\begin{array}{l}0.04 \\
.00 \\
.01 \\
.04 \\
.04 \\
.01 \\
.04 \\
.04\end{array}$ & $\begin{array}{l}116 \\
117 \\
118 \\
119 \\
120 \\
121 \\
122 \\
123\end{array}$ \\
\hline avg d. & - n....... & ………. & & $\ldots$ & . & 2,260 & 580 & 17.6 & 10.4 & 0.58 & 4.35 & $\ldots$ & $\ldots$ & avg. \\
\hline $\begin{array}{l}124 \\
125 \\
126\end{array}$ & $\begin{array}{l}\mathrm{S} \\
\mathrm{S} \\
\mathrm{S}\end{array}$ & $\begin{array}{l}\mathrm{SC} \\
\mathrm{SC} \\
\mathrm{SC}\end{array}$ & & & $\begin{array}{l}\text { Martinsburg, W. Va. } \\
\text { do }\end{array}$ & $\begin{array}{l}19,700 \\
18,600 \\
18,600\end{array}$ & $\begin{array}{l}2,300 \\
1,080 \\
2,290\end{array}$ & $\begin{array}{l}3.4 \\
3.2 \\
3.4\end{array}$ & $\begin{array}{l}2.0 \\
1.5 \\
1.9\end{array}$ & $\begin{array}{r}0.59 \\
.47 \\
.56\end{array}$ & $\begin{array}{l}6.25 \\
6.10 \\
6.25\end{array}$ & $\begin{array}{l}3 \\
2 \\
2\end{array}$ & $\begin{array}{r}0.05 \\
.06 \\
.01\end{array}$ & $\begin{array}{l}124 \\
125 \\
126\end{array}$ \\
\hline $\operatorname{avg}$ & -....... & n.............. & & $\ldots$ & 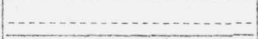 & 19,000 & 1,890 & 3.3 & 1.8 & 0.54 & 6. 20 & ... & …... & avg. \\
\hline $\begin{array}{l}127 \\
128 \\
129\end{array}$ & $\begin{array}{l}\mathrm{C} \\
\mathrm{C} \\
\mathrm{C}\end{array}$ & $\begin{array}{l}\mathrm{SC} \\
\mathrm{SC} \\
\mathrm{SC}\end{array}$ & & & $\begin{array}{l}\text { Baitimore, Md } \\
\text { do }\end{array}$ & $\begin{array}{l}5,300 \\
5,600 \\
3,600\end{array}$ & $\begin{array}{l}780 \\
830 \\
670\end{array}$ & $\begin{array}{l}13.9 \\
13.8 \\
15.5\end{array}$ & $\begin{array}{r}10.2 \\
9.6 \\
11.3\end{array}$ & $\begin{array}{r}0.73 \\
.70 \\
.73\end{array}$ & $\begin{array}{l}4.20 \\
4.10 \\
4.50\end{array}$ & $\begin{array}{l}3 \\
2 \\
2\end{array}$ & $\begin{array}{r}0.03 \\
.11 \\
.03\end{array}$ & $\begin{array}{l}127 \\
128 \\
129\end{array}$ \\
\hline avg ... & 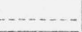 & - & $\ldots$ & & - & 4,800 & 760 & 14.4 & 10.4 & 0.72 & 4. 25 & & -....... & avg. \\
\hline $\begin{array}{l}130 \ldots \ldots \\
131 \\
132\end{array}$ & $\begin{array}{l}\mathrm{C} \\
\mathrm{C} \\
\mathrm{C}\end{array}$ & $\begin{array}{l}\text { DP } \\
\text { DP } \\
\text { DP }\end{array}$ & & & $\begin{array}{l}\text { Alexandria, Va } \\
\text { do do }\end{array}$ & $\begin{array}{l}2,170 \\
4,120 \\
2,500\end{array}$ & $\begin{array}{l}195 \\
270 \\
160\end{array}$ & $\begin{array}{l}18.2 \\
16.6 \\
20.2\end{array}$ & $\begin{array}{l}15.8 \\
14.3 \\
18.8\end{array}$ & $\begin{array}{l}0.87 \\
.86 \\
.93\end{array}$ & $\begin{array}{l}6.25 \\
6.65 \\
6.45\end{array}$ & $\begin{array}{l}3 \\
3 \\
3\end{array}$ & $\begin{array}{r}0.01 \\
.03 \\
.08\end{array}$ & $\begin{array}{l}130 \\
131 \\
132\end{array}$ \\
\hline avg. & & & & ........ & .... & 2,900 & 210 & 18.3 & 16.3 & 0.89 & 6.45 & & & avg. \\
\hline
\end{tabular}

See footnotes at end of table. 
TABLE 6.- Coefficient of thermal expansion of 193 bricks over the temperature range - $10^{\circ}$ to $+40^{\circ} \mathrm{C}$, with description and the data for the other physical tests made on the same bricks-Continued

\begin{tabular}{|c|c|c|c|c|c|c|c|c|c|c|c|c|c|c|}
\hline $\begin{array}{l}\text { Brick } \\
\text { No.a }\end{array}$ & $\begin{array}{l}\text { Mate- } \\
\text { rial }\end{array}$ & $\begin{array}{l}\text { Method of } \\
\text { forming }\end{array}$ & Kiln & $\begin{array}{l}\text { Manufacturer's } \\
\text { grade name }\end{array}$ & Loeality of production & $\begin{array}{l}\text { Comi- } \\
\text { pressive } \\
\text { strength }\end{array}$ & $\begin{array}{l}\text { Moduius } \\
\text { of rup- } \\
\text { ture }\end{array}$ & $\begin{array}{l}\text { 5-hour } \\
\text { boiling } \\
\text { absorp- } \\
\text { tion, } B\end{array}$ & $\begin{array}{c}\text { 24-hour } \\
\text { water ab- } \\
\text { sorption, } \\
C\end{array}$ & $\begin{array}{l}\text { Satura- } \\
\text { tion co- } \\
\text { efficient } \\
(C / B)\end{array}$ & $\begin{array}{l}\text { Coeficient } \\
\text { of thermal } \\
\text { expansion }\end{array}$ & $\begin{array}{l}\text { Num- } \\
\text { ber of } \\
\text { tests }\end{array}$ & $\begin{array}{l}\text { Root mean } \\
\text { square of } \\
\text { deviation } \\
\text { from mean }\end{array}$ & $\begin{array}{l}\text { Brick } \\
\text { No.a }\end{array}$ \\
\hline $\begin{array}{l}133 \\
134 \\
135 \\
136\end{array}$ & $\begin{array}{l}\mathrm{S} \\
\mathrm{S} \\
8 \\
\mathrm{~S}\end{array}$ & $\begin{array}{l}\text { SC } \\
\text { SO } \\
\text { SO } \\
\text { SC }\end{array}$ & & & $\begin{array}{l}\text { Martinsburg, W. V8 } \\
\text { do do } \\
\end{array}$ & $\begin{array}{r}\text { Lb/in. }{ }^{2} \\
14,450 \\
12,500 \\
13,100 \\
14,400\end{array}$ & $\begin{array}{r}\text { Lb/in. } 2^{2} \\
2,100 \\
2,180 \\
2,680 \\
2.500\end{array}$ & $\begin{array}{r}\text { Percent } \\
\text { dry weight } \\
3.5 \\
4.5 \\
6.9 \\
6.4\end{array}$ & $\begin{array}{r}\text { Percent } \\
\text { dryweight } \\
1.5 \\
2.6 \\
5.0 \\
4.6\end{array}$ & $\begin{array}{r}0.43 \\
.58 \\
.72 \\
.72\end{array}$ & $\begin{array}{r}\text { Millionths } \\
\text { per }{ }^{\circ} \mathrm{C} . \\
6.35 \\
6.55 \\
6.20 \\
5.30\end{array}$ & $\begin{array}{l}2 \\
2 \\
2 \\
2\end{array}$ & $\begin{array}{r}\text { Millionths } \\
\text { per }{ }^{\circ} \mathrm{C} . \\
0.03 \\
.02 \\
.04 \\
.01\end{array}$ & $\begin{array}{l}133 \\
134 \\
135 \\
136\end{array}$ \\
\hline $\operatorname{avg} \ldots$ & $\cdots$ & $\cdots \cdots$ & & & … & 13,600 & 2. 360 & 5.3 & 3.4 & 0.61 & 6.10 & $\cdots$ & & avg. \\
\hline $\begin{array}{l}137 \ldots \ldots \\
138 \ldots \ldots \\
139\end{array}$ & $\begin{array}{l}\mathrm{C} \\
\mathrm{C} \\
\mathrm{C}\end{array}$ & $\begin{array}{l}\text { SC } \ldots . . . \\
\text { SO } \\
\text { SO } \ldots . . .\end{array}$ & & & $\begin{array}{l}\text { Columbus, Miss } \\
\text { do do }\end{array}$ & $\begin{array}{l}6,400 \\
6,600 \\
5,300\end{array}$ & $\begin{array}{l}870 \\
900 \\
500\end{array}$ & $\begin{array}{l}16.6 \\
16.8 \\
16.5\end{array}$ & $\begin{array}{l}11.5 \\
12.3 \\
11.8\end{array}$ & $\begin{array}{r}0.69 \\
.73 \\
.72\end{array}$ & $\begin{array}{l}12.40 \\
11.70 \\
10.20\end{array}$ & $\begin{array}{l}3 \\
3 \\
3 \\
3\end{array}$ & $\begin{array}{r}0.08 \\
.06 \\
.10\end{array}$ & $\begin{array}{l}13 i \\
1.38 \\
138\end{array}$ \\
\hline avg ... & ........ & .... & & & $\ldots$ & 6,100 & 760 & 16. 6 & 11.9 & 0.71 & 11.45 & $\cdots$ & …... & avg. \\
\hline $\begin{array}{l}4 \mathrm{~b} \\
55 \mathrm{~b} \\
91 \mathrm{~b} \\
98 \mathrm{~b} \\
116 \mathrm{~b} \\
117 \mathrm{a}^{\mathrm{b}} \\
117 \mathrm{~b}^{\mathrm{b}} \\
120\end{array}$ & & & & 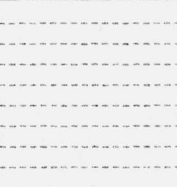 & & & & & |-......... & . & $\begin{array}{l}* 3.2 \\
* 6.0 \\
* 3.4 \\
* 5.3 \\
* 6.1 \\
* 5.3 \\
* 5.4 \\
* 6.9\end{array}$ & $\begin{array}{l}3 \\
2 \\
2 \\
4 \\
2 \\
4 \\
4 \\
2\end{array}$ & $\begin{array}{r}0.17 \\
.10 \\
.17 \\
.08 \\
.24 \\
.02 \\
.06 \\
.26\end{array}$ & $\begin{array}{r}4 \mathrm{~b} \\
55 \\
91 \mathrm{~b} \\
98 \mathrm{~b} \\
1115 \mathrm{~b} \\
117 \mathrm{ab} \\
117 \mathrm{~b} \\
120 \mathrm{~b}\end{array}$ \\
\hline
\end{tabular}

Bricks 19 to 40 are similar to 1 to 22 in the "Water A bsorption of Building Bricks." Am. Soc. Testing Materials 36, pt. 1, 260 (1323). No. 22 is from the same sample as 35 , and there is no brick corresponding to No. 17 or the above report. The remainder of the bricks are in the same order as the that ieport. for

Bereks 05 to 06,07 to $101,106,100$ to 115 , and 119 to 120

120 were cored.

Bricks 67 and 68 are from a different manufacturer, but of similar raw materials to 65 and 66.

Bricks 72 and 73 are of the same type as 69 and 71 , but are not as hard burned. Bricks 79 to 81 are from the same manufacturer as 76 to 78 , but are not as hard burned.
Bricks 87 to 90 are similar to, but not as hard burned as 82 to 86 .
Bricks 91 and 92 are similar to 1 to 5 , but from a shipment received at a much later date. Bricks 93 and 94 are similar to 19 , but from a later shipment. They have a high lime content.

Bricks 97 to 101 are similar to Nos. 26 to 30 in the report "Water Absorption of Buildin Bricks." Am. Soc. Testing Materials 36, pt. 1, 260 (1923).

Bricks 137 to 139 seem to have slag or cinder material mixed with the elay. with the 6-inch gages and data from the other 2-inch gages. The coefficient table.

- Average is for last 4 items only.

a Average is for last 3 items only.

- Average is for last 2 items only. 


\section{EXPERIMENTAL RESULTS}

Table 6 presents the thermal expansions of the 139 bricks used in this investigation, the data from the other physical tests, and a description of the bricks. Bricks 107 and 108 are sand-lime bricks and, while somewhat extraneous to this investigation, are of interest and have been included. Bricks 19 to 40 are similar to those used in an investigation of different methods of making water absorption tests on bricks. ${ }^{6}$ The saturation coefficient is the ratio of the 24-hour water absorption to the 5 -hour boiling absorption (see footnote 4). The number of thermal-expansion tests made on each specimen is given, as well as the standard deviation. The values from the different tests with the 2 -inch gages were weighted, the weight depending on the spread of the four values of the thermal expansion found with each test.

In order to show the methods used in computing the thermal expansion from the tests and other characteristics of the data, more details are given in table 5 for the two tests with the 6 -inch gages on brick 121 and the three tests on brick 122 .

Thermal expansions over a large range of temperature are frequently expressed by a two-constant equation in powers of the temperature, such as

$$
L_{t}=L_{0}\left(1+a t+b t^{2}\right),
$$

in which $L_{0}$ is the length of the specimen at the base temperature, $L_{t}$ is the length at the temperature $t$, and $a$ and $b$ are constants. An effort was made to obtain the second constant, $b$, for some of the bricks by taking a reading at a temperature intermediate between the highest and the lowest. This constant is given in table 4 for the bricks on which two tests gave values which differed by less than $0.002 \times 10^{-6}$.

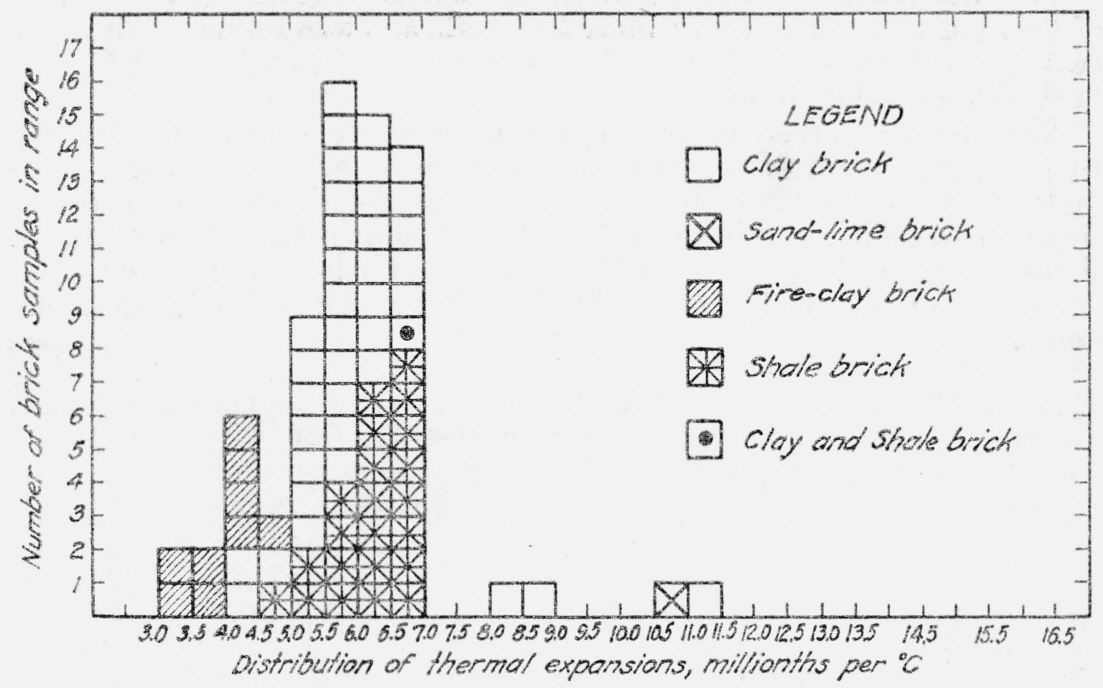

FIQURE 3.-Distribution of the thermal expansions of the clay, shale, sand-lime, and fire-clay bricks.

Figure 3 shows the distribution of the thermal expansions of the bricks The averages were used, rather than the values for the individual

'J. W. McBurney, Water Absorption of Building Brick, Am. Soc. Testing Matexials 36, pt. 1, 260 (1923). 
bricks, in the cases in which more than one brick from the same lot was tested.

In figure 3 it may be noted that the coefficients of thermal expansion of 54 of the 61 lots of clay and shale bricks, or 89 percent, were between 5 and 7 millionths per ${ }^{\circ} \mathrm{C}$. This may be compared with the 22 bricks in the report of the Watertown Arsenal, of which the coefficients for 11 bricks were within this range. Four of the 12 values for clay and shale bricks given by Palmer, and 8 of the 10 for clay and shale tile given by Ingberg and Foster were within the same range. Except for the rather unusual bricks from Whitewater, Wisc. (Nos. 19, 93, and 94) and from Columbus, Miss. (Nos. 137 to 139) the "cut off" at a thermal expansion of 7 millionths per ${ }^{\circ} \mathrm{C}$ for clay and shale bricks is very sharp.

The few values above 7 and below 5 millionths per ${ }^{\circ} \mathrm{C}$ among the clay and shale bricks are chiefly limited to the softer clay bricks (5hour boiling absorption above 14 percent).

The thermal expansions of the fire-clay bricks showed some tendency to decrease with increasing hardness, but the samples are scarcely numerous or representative enough to draw any conclusions on this point.

The thermal expansion of the clay and shale bricks was compared in turn with the compressive strength, modulus of rupture, 5-hour boiling absorption, 24-hour water absorption, and saturation coeffcient. No correlation was found between the thermal expansion and these properties, and, even among bricks from the same shipment, there was no consistent tendency for the thermal expansion to increase or decrease with differences of hardness of burning, as indicated by the above physical properties.

The distribution of the thermal expansions with different methods of forming was studied, but nothing significant was found. The only two types of bricks having coefficients above 7 millionths per ${ }^{\circ} \mathrm{C}$ were both side-cut clay bricks.

The probable error, or rather the probable difference between the thermal expansion of an unknown sample of bricks and the arithmetic mean, has been computed on the basis of the tests reported in this paper and is given in table 7 . The probable error for the clay and shale bricks and the clay bricks is larger, chiefly owing to the large departure of the coefficient for bricks numbered 137 to 139 from the mean.

TABLE 7.-Probable error if the arithmetic mean of the coefficients of thermal expansion is assumed as the coefficient of a brick for which the coefficient is unknown.

\begin{tabular}{|c|c|c|c|}
\hline Types of brick & $\begin{array}{c}\text { Number of } \\
\text { lots }\end{array}$ & $\begin{array}{l}\text { Arithmetic } \\
\text { mean }\end{array}$ & $\begin{array}{l}\text { Probable } \\
\text { error }\end{array}$ \\
\hline & & $\begin{array}{l}\text { Millionths } \\
\text { per }{ }^{\circ} C\end{array}$ & $\begin{array}{l}\text { Millionths } \\
\text { per }{ }^{\circ} \mathrm{C}\end{array}$ \\
\hline $\begin{array}{l}\text { Clay and shale. } \\
\text { Clay and shale with } 5 \text {-hour boiling absorptions less than } 14\end{array}$ & 61 & 6.1 & 0.7 \\
\hline $\begin{array}{l}\text { Clay and shale with 5-hour bolling absorptions less than } 14 \\
\text { percent }\end{array}$ & 32 & 6.0 & 4 \\
\hline Clay & 38 & 6. 0 & .8 \\
\hline Shale & 22 & 6.1 & .4 \\
\hline
\end{tabular}




\section{CONCLUSIONS}

1. The average values of the coefficients of thermal expansion, their range, and the number of samples tested for different types of clay bricks are given in table 8 . For comparison, similar data are given from previous investigations.

TABLE 8.-Average coefficient of thermal expansion (in millionths per ${ }^{\circ} \mathrm{C}$ ) of different types of clay bricks from this and previous investigations

[The values in parentheses are in millionths per ${ }^{\circ} \mathrm{F}$ ]

\begin{tabular}{l|l|l|l}
\hline Types of clays & $\begin{array}{c}\text { Number of } \\
\text { specimens }\end{array}$ & $\begin{array}{c}\text { Average co- } \\
\text { effient of } \\
\text { thermal ex- } \\
\text { pansion }\end{array}$ & $\begin{array}{c}\text { Range of coefficient of } \\
\text { thermal expansion }\end{array}$ \\
\hline
\end{tabular}

PRESENT PAPER (BRICKS) $-10^{\circ} \mathrm{TO}+40^{\circ} \mathrm{C}\left(14^{\circ}\right.$ TO $\left.104^{\circ} \mathrm{F}\right)$

\begin{tabular}{|c|c|c|c|}
\hline $\begin{array}{l}\text { Surface clay } \\
\text { Shale } \\
\text { Fire-clay }\end{array}$ & $\begin{array}{l}80 \\
41 \\
15\end{array}$ & $\begin{array}{l}6.0(3.3) \\
6.1(3.4) \\
3.9(2.2)\end{array}$ & $\begin{array}{l}4.2 \text { to } 12.4(2.3 \text { to } 6.9) \\
4.7 \text { to } 6.8(2.6 \text { to } 3.8) \\
3.0 \text { to } 4.6(1.7 \text { to } 2.6)\end{array}$ \\
\hline
\end{tabular}

PALMER (BRICKS) $-8^{\circ}$ TO $25^{\circ} \mathrm{C}\left(18^{\circ}\right.$ TO $\left.77^{\circ} \mathrm{F}\right)$

\begin{tabular}{|c|c|c|c|}
\hline $\begin{array}{l}\text { Surface clay } \\
\text { Shale } \\
\text { Fire-clay }\end{array}$ & $\begin{array}{l}6 \\
4 \\
4\end{array}$ & $\begin{array}{l}6.6(3.7) \\
4.8(2.7) \\
3.7(2.1)\end{array}$ & $\begin{array}{l}4.7 \text { to } 8.5(2.6 \text { to } 4.7) \\
4.1 \text { to } 5.2(2.3 \text { to } 2.9) \\
3.6 \text { to } 3.8(2.0 \text { to } 2.1)\end{array}$ \\
\hline
\end{tabular}

INGBERG (HOLLOW TILE) $0^{\circ}$ TO $300^{\circ} \mathrm{C}\left(32^{\circ}\right.$ TO $572^{\circ} \mathrm{F}$ )

\begin{tabular}{|c|c|c|c|}
\hline $\begin{array}{l}\text { Surface clay } \\
\text { Shale } \\
\text { Fire-clay }\end{array}$ & $\begin{array}{r}4 \\
6 \\
6\end{array}$ & $\begin{array}{l}6.2(3.4) \\
6.1(3.4) \\
5.5(3.1)\end{array}$ & $\begin{array}{l}5.1 \text { to } 7.3(2.8 \text { to } 4.1) \\
5.7 \text { to } 6.9(3.2 \text { to } 3.8) \\
3.5 \text { to } 6.8(1.9 \text { to } 3.8)\end{array}$ \\
\hline
\end{tabular}

WATERTOWN ARSENAL (BRICKS) $0.6^{\circ}$ TO $100^{\circ} \mathrm{C}\left(33^{\circ}\right.$ TO $212^{\circ} \mathrm{F}$ )

\begin{tabular}{|c|c|c|c|}
\hline Clay and shale ......... & 22 & $6.3(3.5)$ & 3.7 to $13.6(2.1$ to 7.6$)$ \\
\hline
\end{tabular}

2. The coefficients of thermal expansion of 89 percent of the clay and shale bricks were between 5 and 7 millionths per ${ }^{\circ} \mathrm{C}(2.8$ and 3.9 per $\left.{ }^{\circ} \mathrm{F}\right)$, the average being $6.1(3.4)$. The value, 6 millionths per ${ }^{\circ} \mathrm{C}$ (3.3 per $^{\circ} \mathrm{F}$ ), is a good value to assume for the thermal expansion of clay and shale bricks, the probable error, as computed from the data of this paper, being about 0.7 millionth $\operatorname{per}^{\circ} \mathrm{C}\left(0.4\right.$ per $\left.{ }^{\circ} \mathrm{F}\right)$. Of course, if an accurate knowledge of the thermal expansion of any bricks is desired, the above assumption could scarcely be substituted for an actual test.

3. There was no significant difference between the average thermal expansion of the clay and shale bricks. The few values of the coefficient above 7 and below 5 millionths per ${ }^{\circ} \mathrm{C}$ are chiefly limited to the softer clay bricks ( 5 -hr boiling absorption above 14 percent).

4. The thermal expansion of the fire-clay bricks was much lower than that of the clay and shale bricks. The number tested, however, was scarcely large enough, nor were the bricks representive enough to allow definite conclusions to be drawn as to the thermal expansion of other fire-clay bricks. 
5. There was apparently no significant correlation between the thermal expansion and the other physical properties of clay and shale bricks which are customarily measured as tests of quality, and even among bricks from the same shipment there was no consistent tendency for the thermal expansion to either increase or decrease with differences of hardness of burning.

Washington, June 18, 1941. 


\section{MATHEMATICAL TABLES}

Attention is invited to a series of publications which is being prepared by the Project for the Computation of Mathematical Tables conducted by the Federal Works Agency, Work Projects Administration for the City of New York under the sponsorship of the National Bureau of Standards.

To date, six tables have been made available through the National Bureau of Standards. These are listed below:

MT1. Table of the First Ten Powers of the Integers From 1 to 1000:

(1938) VIII +80 pages; heavy paper cover. 50 cents.

MT2. Tables of the Exponential Function $e^{x}$ :

The ranges and intervals of the argument and the number of decimal places in the entries are given below:

$\begin{array}{ccc}\text { Range of } x & \begin{array}{c}\text { Interval } \\ \text { of } x\end{array} & \begin{array}{c}\text { Number of } \\ \text { decimals given }\end{array} \\ -2.5000 \text { to } 1.0060 & 0.0001 & 18 \\ 1.0000 \text { to } 2.5000 & .0001 & 15 \\ 2.500 \text { to } 5.000 & .001 & 15 \\ 5.00 \text { to } 10.00 & .01 & 12\end{array}$

(1939) XV +535 pages; bound in buckram, $\$ 2.00$.

MT3. Tables of Circular and Hyperbolic Sines and Cosines for Radian Argument8:

Contains 9 decimal place values of $\sin x, \cos x, \sinh x$, and $\cosh x$ for $x$ (in radians) ranging from

0 to 2 at intervals of 0.0001 .

(1939) XVII + 405 pages; bound in buckram, $\$ 2.00$.

MT4. Tables of Sines and Cosings for Radian Arguments:

Contains 8 decimal place values of sines and cosines for radian arguments ranging from 0 to 25 at intervals of 0.001 .

(1940) XXIX + 275 pages; bound in buckram, $\$ 2.00$.

MT5. Tables of Sine, Cosine, and Exponential Integrals, Volume I:

Values of these functions to 9 places of decimals from 0 to 2 at intervals of 0.0001 .

(1940) XXVI+ 444 pages; bound in buckram, $\$ 2.00$.

MT6. Tables of Sing, Cosing, and Exponential Integrals, Volumb II:

Values of these functions to 10 places of decimals and 9 or 10 significant figures from

0 to 10 at intervals of 0.001 .

(1940) XXXVII + 225 pages; bound in buckram, $\$ 2.00$.

Payment is required in advance. Make remittance payable to the "National Bureau of Standards", and send with order, using the blank form on the page facing this one for the purpose.

Above prices are for delivery in the United States and its possessions and in countries extending the franking privilege. To other countries the price of MT1 is 65 cents and that of MT2, MT3, MT4, MT5, MT6 is $\$ 2.50$ each remittance to be made payable in United States currency.

Copies of these publications have been sent to various Government depositorie throughout the country, such as public libraries in large cities, and colleges and universities, where they can be consulted.

A mailing list is maintained for those who desire to receive announcements regarding new tables as they become available. A list of the tables it is planned to publish will be sent on request. 